\title{
The Decisive Role of Non-Decision Time for Interpreting Decision Making Models
}

\author{
Gabriel Weindel ${ }^{1,2}$, Thibault Gajdos ${ }^{1}$, Boris Burle ${ }^{2 \mathbb{I I}}$, F.-Xavier Alario ${ }^{1 \mathrm{II}}$ \\ ${ }^{1}$ Laboratoire de Psychologie Cognitive, Aix Marseille Universite, Centre National de la Recherche Scientifique, Marseille, \\ France \\ ${ }^{2}$ Laboratoire de Neurosciences Cognitives, Aix Marseille Universite, Centre National de la Recherche Scientifique, \\ Marseille, France
}

\begin{abstract}
Computational models of decision making account for decision performance by postulating latent cognitive stages, typically distinguishing the durations of decision and non-decision processes. Decision times result from the progressive accumulation of the evidence needed to make a choice. Non-decision times result from the need to encode the stimulus early on, and to generate a response after a decision is reached. The properties of this non-decision time have seldom been investigated. Here we used the onsets of electro-myographical activity in the responding muscles to decompose each reaction time into a pre-motor and a motor time. Drift diffusion models were then fitted on reaction times, and on pre-motor times. This comparison, combined with experimental manipulations of stimulus contrast and response difficulty, provided a window on non-decision processes. The results are best explained by assuming that stimulus encoding does not stop when evidence accumulation starts, and that motor response onset does not always mark the end of the deliberation process, in stark contrast with standard assumptions. This interpretation applies to conditions where participants are asked to favor accuracy over speed, but not vice-versa. Accordingly, the non-decision parameter has to be interpreted differently depending on the participants' adjustments to task instructions. In conclusion, non-decision times reflect cognitively richer processes than is usually assumed, and they can play a decisive constraining role for parameter interpretation in decision making models.
\end{abstract}

\section{Author summary}

Understanding how decisions are made by cognitive agents is a major issue in biology, psychology, psychiatry, or economics. Across these disciplines, a very successful framework, applied to both behavioral and neural data, postulates that decisions result from the progressive accumulation of evidence in favour of one of various options. An additional assumption is that the time it takes to perform a decision includes non-decision processes devoted to stimulus encoding and response execution. The duration of such nondecision processes is seldom studied; it is pointedly referred to as "residual time". Our research shows that overlooking this parameter can compromise the interpretation of other aspects of the model. This is made possible by a physiological approach where part of non-decision durations are measured rather than only estimated.

\footnotetext{
* Corresponding author

E-mail: francois-xavier.alario@univ-amu.fr (F.-XA)

IIBB and F.-XA are Joint Senior Authors
}

\section{Introduction}

Making decisions takes time. In the paradigmatic example of perceptual decisions, where a visual stimulus calls for a motor response, this time is taken by the neural conduction delays from the retina to the brain and from the brain to the muscles (von Helmholtz, 1850, cited by Schmidgen, 2002), and, in between, by the mental action of deciding between alternatives (Donders, 1868). The primary measure used to explore how these decisions are made has been the time elapsing between the stimulation and the participant's overt reaction, or "reaction time" $(R T)$. While $R T$ measures collapse conduction and mental action delays, quantitative processing models have explored the possibility of breaking down $R T$ s into more elementary components.

There is a general agreement that $R T \mathrm{~s}$ can be broken down into at least two parts: a "decision time" $\left(T_{D}\right)$ and a time outside the decision, or "non-decision time" $\left(T_{0}\right)$. The two time periods $T_{D}$ and $T_{0}$ are generally thought to reflect sequential events with additive duration (Ratcliff, 1978; Ratcliff, Smith, Brown, \& McKoon, 2016).

In most decision making models, $T_{D}$ is defined within the evidence accumulation framework, as the time elapsing 


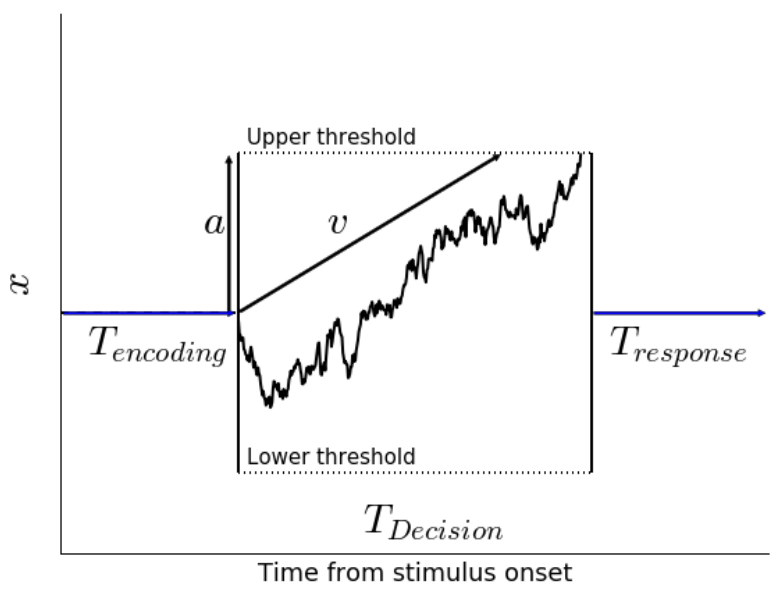

Figure 1. Processing account of a single decision in a diffusion model implementing evidence accumulation.

The stochastic path represents the stimulus evidence being accumulated over time through a noisy channel, modeled as a diffusion process with a drift $v$ (i.e., , rate of accumulation). Accumulation starts at a given amount of evidence (referred to as the bias, figure represents an unbiased decision) and stops once a threshold boundary, a is reached, and the corresponding alternative is chosen. The time between the onset of the accumulation to its termination is referred to as the $T_{D}$, the time before and after, in blue, the $T_{0}$.

from the start of the accumulation of evidence to the time at which the accumulated evidence reaches a decision threshold (Brown \& Heathcote, 2005; Heathcote \& Love, 2012; Ratcliff, 1978; Ratcliff \& Rouder, 1998; Stone, 1960; Usher \& McClelland, 2001, see Stine, Zylberberg, Ditterich, \& Shadlen, 2020, for recent alternatives). $T_{D}$ is computed on the basis of at least three parameters represented in Figure 1: the boundaries (a), usually interpreted as the response caution of the participant, the drift $(v)$, interpreted as the speed of evidence accumulation, and the bias (z), reflecting the starting point of the evidence accumulation process.

$T_{0}$, in turn, is defined as the time needed to form an internal representation of the stimulus $\left(T_{\text {encoding }}\right)$ summed to the time needed to execute the response $\left(T_{\text {response }}\right) . T_{0}$ is often referred to as the "residual" time, a term that reveals the secondary importance given to these aspects of the $R T$ in decision making. Perhaps because of the diagnostic power of $T_{0}$ (e.g., see Smith \& Lilburn, 2020), considerations of this time are generally technical and focused around fitting quality issues rather than functional interpretations. In this paper, we focus on those so-called residual components and show how they shape our understanding of decision making processes.

Formal quantitative models of decision making allow using $R T$ s to test theoretically grounded predictions about cog- nitive processes. So, in theory, manipulating the difficulty of the decision should specifically modulate $T_{D}$ and be reflected in a change of $v$, the drift parameter. This result is indeed observed in several studies (Gomez, Ratcliff, \& Childers, 2015; Palmer, Huk, \& Shadlen, 2005; Voss, Rothermund, \& Voss, 2004). Another example is the manipulation of the speedaccuracy trade-off (SAT), which has been hypothesized to affect response caution, and thus, presumably, to lengthen selectively $T_{D}$ through an adjustment of $a$, the decision threshold. Such is indeed the case (Ratcliff \& McKoon, 2008; Ratcliff \& Tuerlinckx, 2002a), but the SAT manipulation is not always selective, it can also affect the estimation of $T_{0}(\mathrm{Du}-$ tilh et al., 2016; Palmer et al., 2005; Ratcliff, 2006; Voss et al., 2004). Finally, manipulating the color saturation of a visual stimulus or the force needed to produce the response should affect non-decisional input and output processes, respectively. Both manipulations indeed result in a modulation of $T_{0}$ (Gomez et al., 2015; Ho, Brown, \& Serences, 2009; Servant, White, Montagnini, \& Burle, 2016; Voss et al., 2004); however, they also impact the parameters determining $T_{D}$ : brightness manipulations affect $v$ (Servant et al., 2016), force manipulations affect $z$ (Voss et al., 2004) and $a$ (Gomez et al., 2015; Ho et al., 2009).

In other words, experimental manipulations are not always reflected in modulations of the parameters predicted by the theory. This discrepancy may receive two alternative explanations: either, the model does not appropriately implement the theory, or the theory itself (i.e., the psychological interpretation of the experimental manipulations) is incorrect. A clarification of this issue is required to better ground the theoretical interpretation of the parameters, especially in the context of an increasing reliance on the DDM to address both basic and clinical research questions.

\section{The present study}

Our research on the links between parameters and processes in decision making models focused on the "residual" non-decision time $T_{0}$ estimated in the Drift Diffusion Model (DDM, Ratcliff \& Tuerlinckx, 2002b). To test how faithfully $T_{0}$ reflects the cognitive delays it is intended to capture (i.e., $T_{\text {encoding }}$ plus $T_{\text {response }}$ ), we used targeted experimental manipulations coupled with empirical response time decomposition in a perceptual decision task.

The $R T$ s of every trial were decomposed into pre-motor and motor times (PMT and $M T)$, based on muscle activity onsets revealed by the electromyogram (EMG) of the responding hand (Figure 2a; see also Burle, Possamaï, Vidal, Bonnet, \& Hasbroucq, 2002). Following Luce (1986, p. 97) and our own previous work (Weindel, Anders, Alario, \& Burle, 2021), we assume that $M T$ measures at least a sub-set of the time allocated to response execution, $T_{\text {response }}$. It follows from this assumption that $M T$ should be well captured by the $T_{0}$ obtained when fitting the DDM to $R T \mathrm{~s}$. It also 
follows from the assumption that the remainder PMT should primarily correspond to the sum of $T_{\text {encoding }}$ and $T_{\text {decision }}$, excluding most of $T_{\text {response }}$. The DDM can thus be fitted either to RTs, to estimate $T_{0}$ as is usually done, or be fitted to PMTs, to provide a reasonable estimate of $T_{\text {encoding. }}$. These assumptions are made explicit to guide our interpretations. Their appropriateness will be examined in the General Discussion in light of the observed results.

The fractionation of $R T$ into $P M T$ and $M T$ proposed in the previous paragraph is not always straightforward. On some trials, substantial EMG activity is present at multiple time points throughout the stimulus-response interval (Figure 2b; see also Weindel et al., 2021). These multiple EMG bursts may reflect genuine response hesitations, alternative response strategies (e.g., mind wandering, partial guessing), artifacts (e.g., motor noise or failure to deploy enough force to trigger the end of the trial), etc. Such trials would need additional assumptions to be interpreted. To stick to the serial encoding, decision, and motor stages postulated in canonical evidence accumulation models, trials with multiple EMG bursts were excluded from the core analysis of the current study (see Servant, Logan, Gajdos, \& Evans, 2021, for an alternative modelling strategy).

In addition to the EMG decomposition, there were three experimental manipulations. The two first ones were intended to primarily affect $T_{0}$ : a manipulation based on stimulus contrast was predicted to affect the process of encoding, and a manipulation of the response force requirements was predicted to affect the process of responding. Finally, the participants' strategy was manipulated with a classic SpeedAccuracy trade-off. The details for these three manipulations are as follows.

Affecting encoding processes. Participants had to choose which of two sinusoidal gratings had the highest contrast. We manipulated the mean contrast of the gratings, while keeping their absolute difference constant. It has been shown that stimuli become more accessible, and are processed faster, when their contrast increases (e.g., Harwerth \& Levi, 1978, in a simple reaction time task). We thus expected shorter $T_{\text {encoding }}$ for higher contrasts. Conversely, WeberFechner's law (Stevens, 1961) states that the just noticeable difference between a pair of stimulus increases linearly with stimulus intensity. We thus expected that discriminating between the two gratings will be more difficult for higher contrasts, thereby inducing longer $T_{D}$ through a decrease in the drift parameter (for a detailed account on how such manipulation can affect the $T_{D}$ see Ratcliff \& McKoon, 2018). In sum, the contrast manipulation was expected to have opposite effects on $T_{\text {encoding }}$ and on $T_{D}$.

Affecting motor processes. We manipulated the force required to produce the responses, a manipulation known to affect processes related to motor execution measured by $M T$ (Burle et al., 2002). We expected that an increase in the re- quired response force will result in an increase of $T_{\text {response }}$. This would be consistent with studies where similar response output manipulations resulted in changes in the estimated $T_{0}$ parameter of the drift diffusion model (Gomez et al., 2015; Ho et al., 2009; Voss et al., 2004). Conversely, the $T_{0}$ fitted on PMT (i.e., putatively $T_{\text {encoding }}$ ) was not expected to be affected by the Force manipulation. It has occasionally been reported that force requirements can also influence the decision related parameters of bias and threshold (Gomez et al., 2015; Voss et al., 2004), but no explicit interpretation has been offered. Our EMG decomposition and the hypothesized absence of decision-related latency variations in $M T$ allows the following subsidiary expectations. If the force manipulation genuinely affects the decision processes, the effect should be invariant whether the fit is performed on RT or on PMT. Conversely, observing that the effects vary between both fits would suggest an incorrect separation of $T_{D}$ and $T_{0}$.

Speed accuracy trade-off (SAT). Finally, we manipulated the SAT level required from participants through verbal instructions. This manipulation is classically described as an adjustment of the level of evidence needed before a decision is made, and is therefore linked to a change in the boundary parameter of the DDM. However, the manipulation of SAT has also been shown to modulate the speed of encoding processes (Steinemann, O'Connell, \& Kelly, 2018) and of motor execution (Spieser, Servant, Hasbroucq, \& Burle, 2017; Steinemann et al., 2018; Weindel et al., 2021). There is a debate between accounts of SAT, either in terms of parameter (i.e. process modulations) within DDM or in terms of changes in the nature of the generative model (e.g. Cisek, Puskas, \& El-Murr, 2009; Dutilh, Wagenmakers, Visser, \& van der Maas, 2011; Ollman, 1966; Verdonck, Loossens, \& Philiastides, 2021)a. Here, decision instructions were manipulated to test whether the above derived predictions on encoding and motor processes hold across the SAT spectrum.

\section{Results}

\section{General overview of findings}

Before detailing the results, we first present an overall summary to guide the reader. Replicating multiple previous observations, the manipulation of SAT instructions resulted in an adjustment of both the decision boundary parameter $a$ and $T_{0}$. Unexpectedly, however, the effect of the manipulation of force and contrast on the DDM parameters depend on the SAT instruction. When instructions emphasized the speed of the responses, the manipulation of contrast translated into the expected effects on $T_{0}$ and on the drift rate, both when the DDM was fitted on $R T$ and on $P M T$. The manipulation of force specifically impacted $T_{0}$, only when estimated from $R T$, while leaving decision related parameters unchanged. Conversely, when the instructions emphasized the accuracy of the responses, the expected decrease in $T_{0}$ 

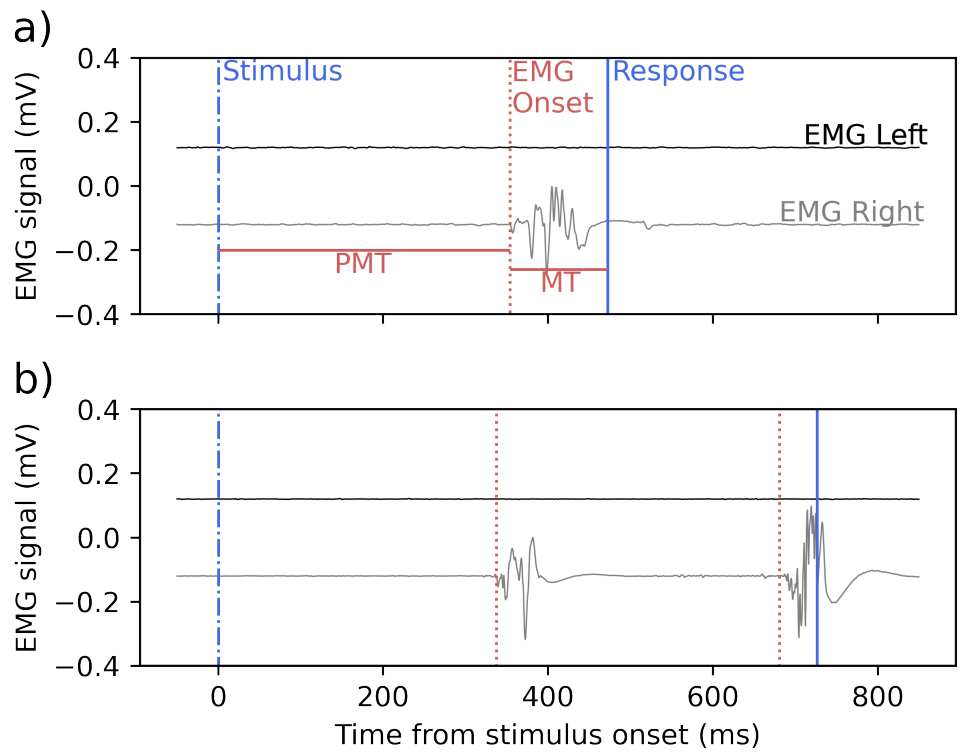

Figure 2. Two examples of the EMG time courses of each hand within a trial, adapted from Weindel et al. (2021). a) Example of the decomposition of a single RT into pre-motor and motor times based on the onset of the unique EMG burst leading to a response.

b) Example of a trial with multiple EMG bursts where the first burst did not lead to a mechanical response

with increasing contrast was absent. The force manipulation did have an unexpected effect on decision related parameters (boundary) particularly when DDM was fitted on PMT.

\section{Data pre-processing}

The method implemented for detecting EMG onsets imposed an $R T$ upper limit of $1500 \mathrm{~ms}$, whereby $1 \%$ of the trials were excluded. Trials with low signal-to-noise ratio or with high spontaneous tonic activity that resulted in uncertain EMG onset detection were excluded (7\%). In agreement with our assumption of EMG partition, trials that presented more than one EMG activity (see Figure 2b) were also excluded $(21 \%)$. Finally, the trim criterion derived from the fast-guess detection method (see Method section) lead to the exclusion of $8 \%$ of the data. Thus, the combined EMG and statistical criteria resulted in the exclusion of $37 \%$ of the trials. This proportion may seem high, so it is important to highlight that these exclusions are not due to participants' overt errors, but for the most part to our a priori hypothesis about the interpretability of EMG bursts. Censoring errors, for the LMM analysis of RT, PMT, and MT, removed 13\% of the remaining data. On average, 1513 trials $(\mathrm{SD}=310)$ were available per participant. All estimated differences $(\hat{d})$ are presented on the data scale, milliseconds for chronometric variables and proportion correct for accuracy. A replication of the results on DDM modelling on $R T$ s with another dataset is presented in Appendix $\mathrm{C} 1$.

\section{Behavioral results ( $R T$ and error rates)}

Linear mixed models. The following observations are illustrated in Figure 3 and in Figure 4 (two left columns). When speed is emphasized, $R T$ decreases, $\hat{d}_{S A T}=-165$, $C r I=[-197,-134]$, and so does the accuracy rate, $\hat{d}_{S A T}=$ $-0.10, C r I=[-0.13,-0.07]$. The effect of contrast on $R T$ proved to be different between SAT instructions $\hat{d}_{\text {Contrast:S peed-Acc. }}=-55, C r I=[-70,-39]$. When participants are asked the emphasize accuracy, an increase in contrast lengthens $R T \mathrm{~s}, \hat{d}_{\text {Contrast: } A c c .}=60, C r I=[35,83]$, and reduces response accuracy, $\hat{d}_{\text {Contrast:Acc. }}=-0.22, \mathrm{CrI}=$ $[-0.27,-0.18]$. The contrast effect is essentially canceled when participants are asked to speed their responses, $\hat{d}_{\text {Contrast:S peed }}=5, C r I=[-13,22]$. The proportion of correct responses however displayed similar effects of Contrast in both the Speed and Accuracy conditions, $\hat{d}_{\text {Contrast:Speed }}=$ $-0.22, C r I=[-0.27,-0.16], \hat{d}_{\text {Contrast:S peed-Acc. }}=0.02$, $C r I=[-0.01,-0.04]$.

When force requirements are higher, $R T$ increases both in the Accuracy, $\hat{d}_{\text {Force:Acc. }}=48, C r I=[22,75]$ and the Speed conditions, $\hat{d}_{\text {Force: } \text { peed }}=35, C r I=[16,53]$. The proportion of correct response is not affected by the Force factor neither in accuracy $\hat{d}_{\text {Force:Acc }}=0.01, C r I=[-0.01,0.02]$ nor in the speed condition $\hat{d}_{\text {Force }: \text { peed }}=0.03, \mathrm{CrI}=[0.00,0.05]$ although the CrI barely included 0 .

Unexpectedly, the interaction between Force and Contrast had an effect on $R T$ selectively in the Accuracy condition, 

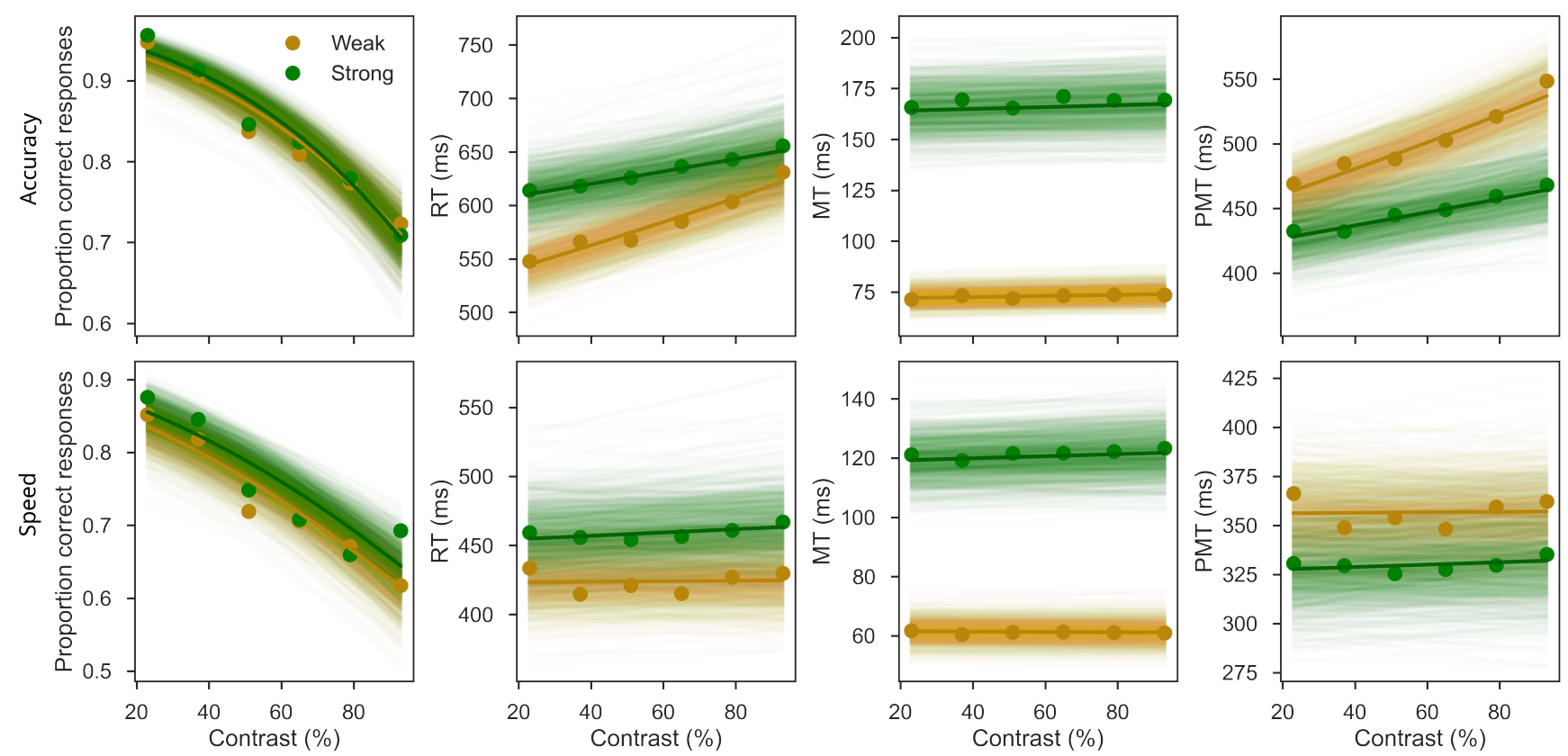

Figure 3. Average values for proportion correct, $R T, M T$, and $P M T$ (columns from left to right) plotted for each SAT condition (accuracy on the top row and speed on the bottom row), broken down by contrast levels and by force condition. The lines represent 1000 random draws from the joint posteriors of the combined MCMC chains of the corresponding G/LMM fits. The thick line represent the predicted regression line with all parameters set at their maximum a posteriori value.

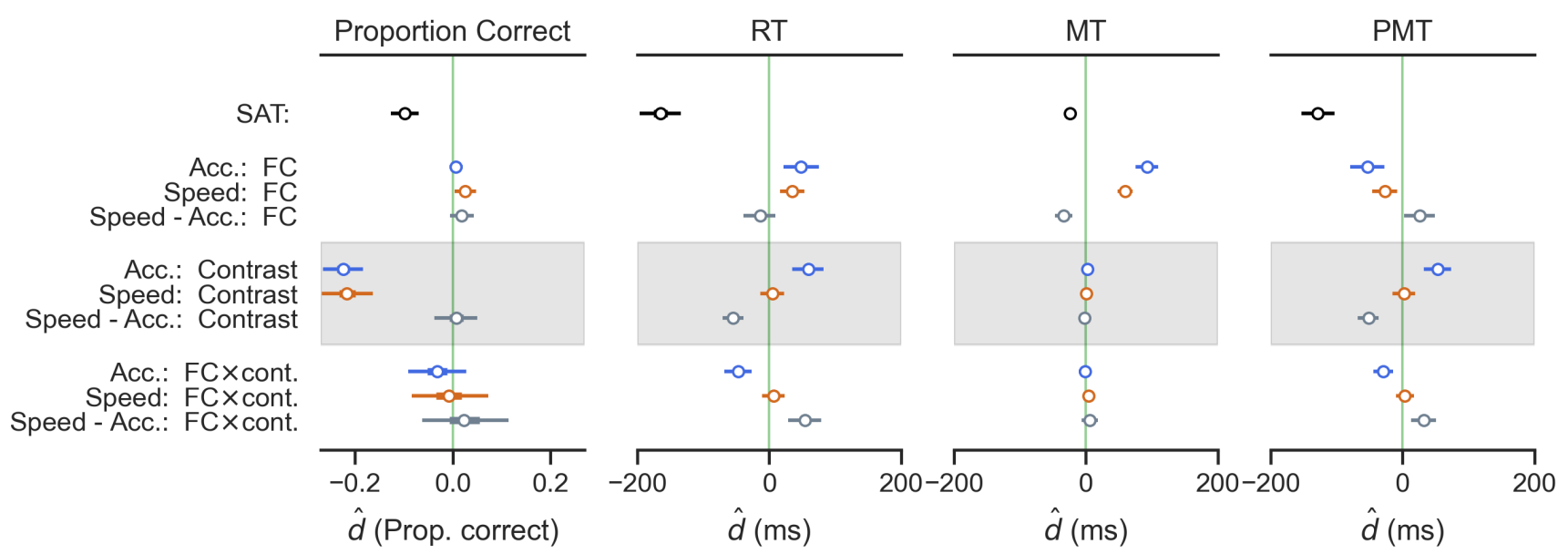

Figure 4. Estimated differences between condition levels $(\hat{d})$ for SAT, Force (FC), Contrast and their interactions on the millisecond scale of the data in the Accuracy condition (blue), the Speed condition (chocolate), and the difference between both conditions (i.e. interaction; grey). Dots represent the maximum a posteriori, and bars the $2.5 \%$ and $97.5 \%$ HPD of the corresponding marginal posterior distributions.

$\hat{d}_{\text {Force } \times \text { Contrast:Acc. }}=-47, \mathrm{CrI}=[-68,-27]$ but not in the Speed condition $\hat{d}_{\text {Force } \times \text { Contrast: Speed }}=7, \mathrm{CrI}=[-11,23]$. The proportion of correct responses was not sensitive to the interaction between force and contrast neither in Accuracy $\left.\hat{d}_{F o r c e \times C o n t r a s t: A c c}=-0.03, C r I=[-0.09,0.03]\right)$ nor in Speed, $\hat{d}_{\text {Force } \times \text { Contrast: Speed }}=-0.04, C r I=[-0.08,0.07]$.
Drift Diffusion Model selection on RT. The model selection procedure is fully described in Appendix B, with DIC and BPIC estimates summarized in Table B1. In the model that was ultimately selected, one boundary parameter was estimated for each combination of SAT and force condition levels, one drift for each level of contrast, one starting point for each force level, and one non-decision time for each experimental cell of the three factors SAT $\times$ Force $\times$ Con- 

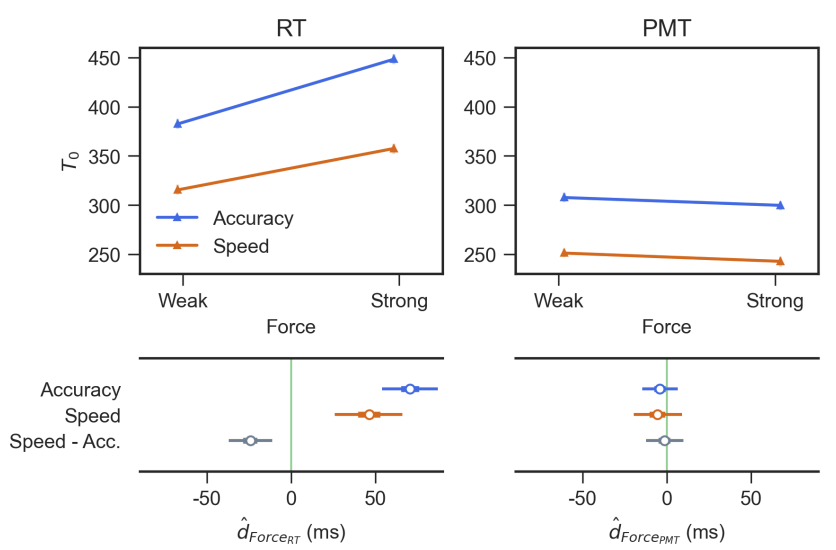

Figure 5. Effect of Force on the parameter $T_{0}$ estimated on RTs (left panel) vs. on PMTs (right panel).

Upper row: estimated mean values and one SD as shaded lines (barely visible).

Bottom row: posterior distribution of the DDM parameter for the effect of Force on $T_{0}$ at each level of SAT, and of its interaction with SAT (see $T_{0} \mathrm{SAT} \times \mathrm{FC}$ in Table 1 ).

trast (see Table B1). The effects of the experimental factors on these model parameters are summarized in Table 1 and spelled out below.

Effects on $T_{0}$ when fitted on RT. Estimated $T_{0}$ was longer when accuracy was emphasized. SAT and force interacted: increasing force had a strong effect on $T_{0}$ in the accuracy condition, and the interaction term indicated a smaller yet reliable effect in the speed condition (see left column of Figure 5). SAT and Contrast also interacted (see left column, middle and bottom panels, of Figure 6): there was no evidence for an effect of contrast on $T_{0}$ in the accuracy condition, but the interaction term indicated a negative effect when speed was emphasized. Finally, there was no evidence for an interaction between Force and Contrast, nor for the three way interaction (although in this latter case the CrI barely included 0). These estimates are summarized in Table 1.

Effects on decision related parameters when fitted on RT. The model selection procedure revealed that the drift rate was affected by Contrast (see left column top panel of Figure 6 and Table 1), but not by SAT nor Force (for details, see model selection results in Table B1). The boundary parameter was affected by SAT, being smaller for the speed condition, and it was not reliably affected by Force in either SAT condition (see left panel in Figure 7). The starting point parameter revealed no evidence for an effect of Force (with the provision that the corresponding CrI barely included 0 ; Table 1).

Summary and discussion of behavioral observations and the DDM fit on RT. Regarding the targeted encoding processes, performance decreased with increasing contrast.

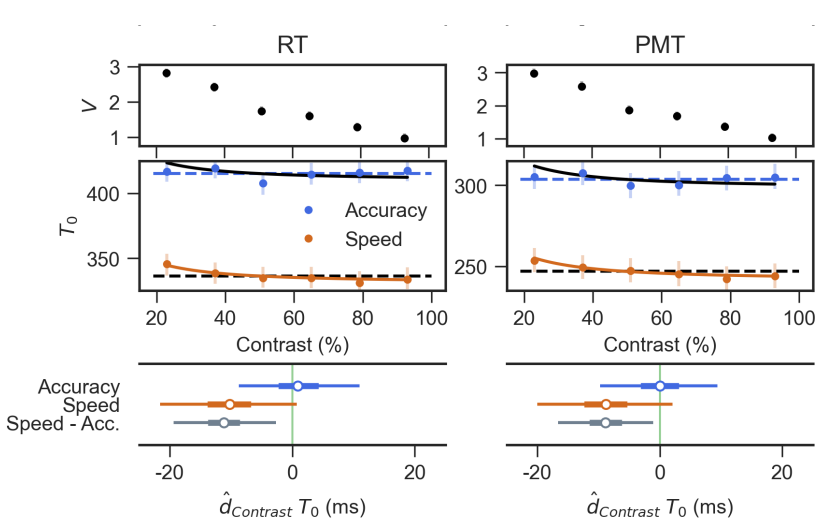

Figure 6. Effect of Contrast on the parameters drift rate $(V)$ and $T_{0}$ estimated with DDM on RT (right column) and PMT (left column) in the speed and accuracy conditions.

Panels in the top row show the estimated drift rate $(V)$ mean values and one SD as shaded lines along with the contrast levels.

Panels in the middle show the estimated $T_{0}$ mean values and one SD as shaded lines. In the General Discussion section we describe external data whose fit is represented by the plain line and compared to the mean $T_{0}$ of each condition represented by the dashed lines. The best fit, as assessed using an $R^{2}$, between both lines is colored according to the color code of the condition.

Panels in the bottom row show the posterior distribution of the effect of contrast in accuracy and speed along with the difference between both conditions $\left(T_{0} \mathrm{SAT} \times \mathrm{FC}\right.$ in Table 1 and in the replication Table D2).
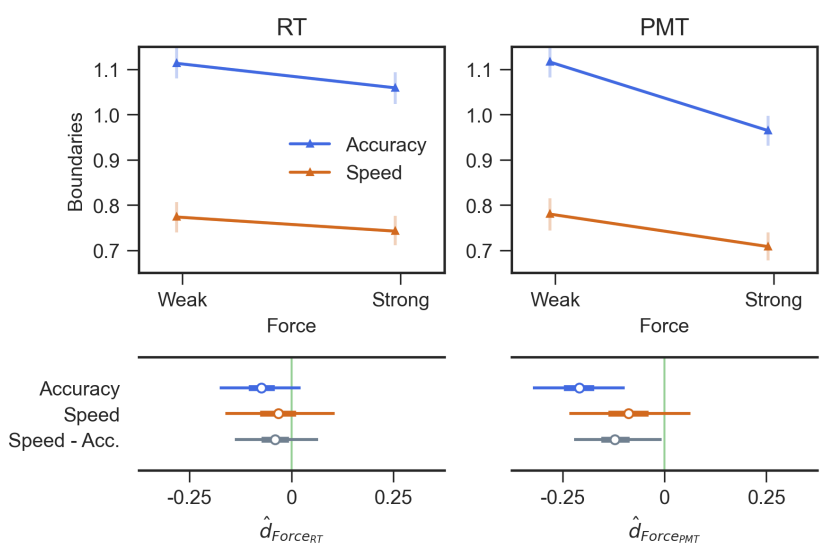

Figure 7. Effect of Force on the boundary parameter a when estimated on RTs (left panel) vs. on PMTs (right panel).

Upper row: estimated mean values and one SD as shaded lines.

Bottom row: posterior distribution of DDM regression coefficients for the effect on $a$ of Force in accuracy, speed and their difference on either chronometric variable (see Boundaries in Table 1). 
This variation was captured by the model as the predicted negative relation between contrast and $T_{0}$, as well as the predicted negative relation between contrast and drift - the latter resulting in a positive relation between contrast and $T_{D}$. However, the effect of Contrast on $T_{0}$ was only present when participants were asked to emphasize speed over accuracy. Because $T_{0}$ aggregates $T_{\text {encoding }}$ and $T_{\text {response }}$, which cannot be estimated separately, a post-hoc account of the unexpected absence of Contrast effect in the Accuracy condition would be to hypothesize opposite effects on the two components of $T_{0}$. We show in the next sections on $M T$ and $P M T$ measures that this account is very unlikely.

Regarding the targeted motor processes, $R T$ increased with higher Force demands, while the rate of correct responses was not affected by Force. The variation in $R T$ s was captured by the DDM as a rather selective increase in $T_{0}$ that left all other parameters unaffected (with the possible exception of bias). However, there was a discrepancy between the effects of Force estimated on $R T$ vs. on the $T_{0}$ fitted values. In the $R T$ analysis, the effect size of Force was $48 \mathrm{~ms}$ in the accuracy condition and $35 \mathrm{~ms}$ in the speed condition, and Force did not interact with SAT; in the $T_{0}$ fitted values, Force interacted with SAT and its values for the Accuracy and Speed conditions were $71 \mathrm{~ms}$ and $46 \mathrm{~ms}$, respectively. In the next section, we quantify factor effects on $M T$ and we build on our assumption linking $M T$ to $T_{\text {response }}$ to assess whether the more faithful capture of motor processes comes from linear regression on $R T$ or from DDM fitting.

Regarding the parameters affecting the decision time $T_{D}$, the observed effects were exactly as predicted: the drift was selectively affected by the Contrast manipulation and the boundary was selectively affected by the SAT manipulation. All effects found on the DDM parameters fitted on RT are replicated in a different dataset to the exception of the interaction between SAT and Force on $T_{0}$ (see Appendix C).

\section{Motor times $(M T)$}

Linear mixed model. The following observations are illustrated in Figure 3 and in Figure 4 (third column). The SAT condition Speed reduced $M T, \hat{d}_{S A T}=-24, C r I=$ $[-30,-17]$. A higher force requirement increased $M T$, both in the accuracy, $\hat{d}_{F C: A c c}=93, C r I=[75,110]$, and the speed conditions, $\hat{d}_{F C: S \text { peed }}=59, C r I=[48,71]$. The interaction term confirmed that the effect of Force was indeed smaller when speed was emphasized $\hat{d}_{F C: S \text { peed-Acc. }}=-34$, CrI $=[-47,-20]$.

Contrast and its interaction with Force had no reliable effect on MT across any of the SAT conditions, as indicated by coefficients restricted to low effect sizes and $\mathrm{CrIs}$ containing 0 .

Summary and discussion of $M T$ results. We found no evidence for an effect of contrast on $M T$ nor of its interaction with SAT. In the section on behavioral results ( $R T$ and error rates), we reported an interaction between Contrast and SAT, and speculated that Contrast may have opposite effects on $T_{\text {encoding }}$ and $T_{\text {response }}$, the two components of $T_{0}$, in the Accuracy condition. The current analysis of $M T$ shows that this hypothesis is implausible. One alternative possibility is that response mechanisms in the Speed and Accuracy conditions are different enough that they are differently sensitive to the encoding of contrast. We come back to this issue in the General Discussion.

The expected effect of Force on $M T$ was clear, but its size (93 $\mathrm{ms}$ and $60 \mathrm{~ms}$ in the Accuracy and Speed conditions, respectively) was much larger than that observed on $R T$ (48 ms and $35 \mathrm{~ms}$ ). Because each $R T$ is the sum of its corresponding $M T$ and $P M T$, this discrepancy can only be explained by an opposite effect of Force on $P M T$. We pursue this issue in the next section on $P M T$.

The effect size of Force on $M T$ was also remarkably higher than the effect size estimated in the previous section on $T_{0}$ (71 and $47 \mathrm{~ms}$ ). Our assumption linking $M T$ to the $T_{\text {response }}$ component of $T_{0}$ invites, again, a tentative compensation account in which the other component of $T_{0}$, namely $T_{\text {encoding, }}$, would be sensitive to Force in the opposite direction. While this hypothesis may seem counter-intuitive, it can be directly tested in our framework by fitting $D D M$ to $P M T$ distributions, as we do in the next section.

\section{Pre-Motor times $(P M T)$}

Linear Mixed Model. The results for PMT were very similar to those reported above for $R T$ with the important exception of the effects of Force. This can be appreciated by comparing the second and fourth columns in Figure 3 and Figure 4. The effect of Force on PMT was opposite to that observed on $R T$ both in accuracy, $\hat{d}_{F C: A c c}=-53$, $C r I=[-79,-27]$ and speed conditions, $\hat{d}_{F C: \text { sped }}=-27$, $C r I=[-46,-8]$. Here the effects of Force were reliably different across SAT conditions, $\hat{d}_{F C: S \text { peed }-A c c}=26$, $C r I=[2,50]$.

Drift Diffusion Model selection. The model selection procedure applied to $P M T$ selected the same model structure that was selected when the procedure was applied to $R T$, namely M13 in Table B1.

Effects on $T_{0}$ when fitted on PMT. The patterns of effects were similar for both $P M T$ and $R T$ fits, with the following important exceptions (compare the two panels on Table 1). The main difference is that neither Force nor its interaction with SAT appeared to affect the $T_{0}$ estimated on $P M T$ (Figure 5 right panel). In addition, we highlight in Figure 6 that the effect of contrast on $T_{0}$ interacted with SAT. The effect was in the expected direction in the Speed condition (although the CrI included 0) but centered on 0 in the Accuracy condition. 
Effects on decision related parameters when fitted on PMT. Contrary to the results observed with a fit on $R T$, in the fit on $P M T$ the boundary parameter was affected by Force and this effect interacted with SAT. Increasing Force resulted in a lower boundary parameter, an effect that was much reduced (if not absent) in the Speed condition (Figure 7 right panel). The other decision parameters, drift and bias, were roughly similar when derived from fits on $P M T$ and $R T$ (compare the two panels on Table 1).

Summary and discussion of $P M T$ observations and their DDM fit. The effects of Contrast were consistent with those observed on $R T$, revealing "opposite" effects on $T_{0}$ and drift, including the fact that Contrast interacts with SAT instructions showing that the expected effect is mainly present in the Speed condition only (Figure 6). PMT does not include $M T$, which we hypothesized to be strongly linked to $T_{\text {response }}$. Therefore, in this analysis, $T_{0}$ provides a reasonable estimate of $T_{\text {encoding. }}$. In sum, encoding processes are orderly affected by Contrast in the Speed condition only.

Conversely, the effect of Force was remarkably different for $R T$ and for $P M T$. The linear models revealed Force effects of opposite signs on $R T$ and $P M T$, whereby $P M T$ s were shorter with stronger force (Figure 3). The DDM fit of $P M T$ did not capture this effect on $T_{0}$ (Figure 5). This absence of effect is consistent with the assumption that, here, $T_{0}$ indexes force-independent encoding processes (i.e. $T_{\text {encoding }}$ ). It undermines the tentative hypothesis (from the section on $M T$ ) that opposite effects of Force compensate one another on the two components of $T_{0}, T_{\text {encoding }}$ and $T_{\text {response }}$ Instead, the DDM fit attributed the effect of Force to the boundary parameter. Boundary decreased with increasing Force requirements, in the Accuracy condition only (Figure 7). This means that the DDM fits on $R T$ and on $P M T$ lead to different attributions of the Force effect, particularly in the Accuracy condition.

\section{General Discussion}

The reported findings help clarifying how faithfully a quantitative model such as DDM can separate decision and non-decision processes. In this General Discussion, we consider the consequences of observing that the response output manipulation changed decision related parameters, in contrast to common expectations. We then re-examine the fact that $T_{0}$ did not conform to the predictions regarding visual encoding processes in the accuracy condition. We arrive at the general conclusion that observing different findings across SAT conditions challenges the assumed drift diffusion generative model, despite the rather canonical experimental setting we used.

\section{$T_{0}$ and motor processes}

The common assumption that $T_{0}$ contains processes related to motor execution or, the more specific assumption we used in this study that $T_{\text {response }}$ is equal to $M T$ (Luce, 1986; Weindel et al., 2021), entails two predictions. The first one is that $T_{0}$ estimated using a DDM fitted on $R T$ should be sensitive to the force manipulation whereas $T_{0}$ fitted on $P M T$ should not. As expected $M T$ (measured) and $T_{0}$ (estimated) are both influenced by the force required to respond. We found no effect of force on $T_{\text {encoding. }}$. This pattern of results strengthens the hypothesis that $T_{0}$ is the sum of two components, one of which captures motor processes.

The second prediction is that decisional parameters should be the same when estimated on $R T$ vs. PMT. This is because motor processes are hypothesized to start after the threshold has been reached. This prediction proved inaccurate, most clearly in the condition combining high force condition and accuracy; there, the threshold parameter was lower in the $P M T$ than in the RT DDM fits (Figure 7). The observations in this condition can be accounted for if the EMG onset does not index the end of the accumulation process estimated by DDM.

If the standard fit on $R T$ is taken as a reference to establish decision duration, then the shorter decision durations estimated based on PMT invites the inference that the decision process (i.e., accumulation of evidence) continues beyond EMG onset. In other words, the appropriate interpretation of the response component of $T_{0}$ (i.e., $T_{\text {response }}$ ) is it that starts as late as after EMG onset. Evidence that the decision process is pursued during response execution has previously been observed (Buc Calderon, Dewulf, Gevers, \& Verguts, 2017; Resulaj, Kiani, Wolpert, \& Shadlen, 2009; Selen, Shadlen, \& Wolpert, 2012). Crucially, the response settings in those previous studies were very different, e.g., pointing or reaching movements, and response execution took much longer than for our (isometric) button presses (for a related discussion see Burle, Roger, Vidal, \& Hasbroucq, 2008; Scaltritti, Job, Alario, \& Sulpizio, 2020).

\section{$T_{0}$ and encoding processes}

We observed, as expected, a negative relation between contrast and non-decision processes whether estimated on the whole $R T\left(T_{0}\right)$ or the PMT ( $\left.T_{\text {encoding }}\right)$. Unexpectedly, this was only true when participants had to respond rapidly, but not when accuracy was emphasized. We undertook additional analysis to better understand this discrepancy.

Latencies related to the encoding of visual gratings have been found to be negatively and non-linearly related to stimulus contrast in psychophysical (Harwerth \& Levi, 1978) and neurophysiological (Reynaud, Masson, \& Chavane, 2012) studies. This well established effect concerns the early $(\sim$ $80 \mathrm{~ms}$ ) discharge latency of V1 neurons in macaques. Such 
an early process is not expected to be modulated by response strategy adjustments such as SAT. Therefore, if our estimated $T_{\text {encoding }}$ reflects these stages, it should be non-linearly related to the contrast manipulation similarly across SAT levels.

This prediction was tested against external data from Reynaud et al. (2012). These authors measured the latency of the onset of cortical neuron activity in visual area V1 in awake monkeys (described in Appendix D1). Their data provide onset times of V1 neurons, revealing a non-linear increase of onset-latencies across visual gratings of decreasing contrast. Reynaud et al. (2012) then used these latencies to fit a well established neurophysiologically motivated model (Naka \& Rushton, 1966) using the inverted equation as in Barthélemy, Fleuriet, and Masson (2010) (summarized in Appendix D). If $T_{\text {encoding }}$ really reflects the low level extraction of stimulus features, its latency should follow the same quantitative relationship. We tested whether the modulation of our estimated $T_{\text {encoding }}$ matches the modulation observed in V1 (Reynaud et al., 2012) by calculating an $R^{2}$ across contrast levels between the centered V1 data and our centered $T_{\text {encoding, }}$, broken down by SAT and force conditions (see Figure 6 and Figure D1 in Appendix D for a close-up).

In the condition where speed is emphasized and the force required is weak, there was an almost perfect adjustment $\left(R^{2}=0.99\right)$ between the model fitted on V1 discharge latencies and our out-of-sample $T_{\text {encoding }}$ estimates. When speed is emphasized but the force required is high, we observed a lower but still substantial agreement $\left(R^{2}=0.63\right)$. Thus, under speed instructions, $T_{\text {encoding }}$ likely reflects a meaningful duration pertaining to visual processing.

In contradistinction, the model fitted on V1 discharge latencies did not adjust to $T_{\text {encoding }}$ when accuracy was emphasized, neither in the low nor the high force conditions $\left(R^{2}=-0.71, R^{2}=-1.14\right.$, respectively $){ }^{1}$ Thus, under accuracy instructions, the variations of $T_{\text {encoding }}$ are not easily linked to variations of early visual process durations.

\section{Ideas and Speculation}

In addition to modifying the standard interpretation of $T_{\text {response }}$, our results questions the interpretability of EMG onsets in the context of DDM. An EMG onset occurring during the evidence accumulation process would challenge our hypothesis that $P M T=T_{\text {encoding }}+T_{D}$. Other aspects of the data help clarifying this issue. Goodness of fit was comparable for $P M T$ and $R T$ fits. $P M T$ could thus be generated by a diffusion process. Moreover, the other decision parameters (drift and bias) were essentially similar across both fits. This would happen if the EMG onset and the end of $T_{D}$ corresponded to the crossing of two different thresholds for the same diffusion process. Such double-threshold hypothesis is congruent with a recent theory proposing that motor execution is determined by an evolving decision variable (Servant et al., 2021; see also Burle et al., 2008; Servant, White, Montagnini, \& Burle, 2015).

On the other end, the lack of evidence of the link between $T_{\text {encoding }}$ and early visual process durations when accuracy is emphasized is congruent with a recent finding which shows, using electro-encephalographic data, an additional pre-decision stage specifically in the accuracy condition (van Maanen, Portoles, \& Borst, 2021). This additional stage could absorb the effect of contrast, in the spirit of a cognitive slack account (Oriet \& Jolicœur, 2003; Pashler, 1994). This qualitative modulation driven by the participants' strategical adjustments to the SAT instructions suggests that "cognitive" processes occur prior to evidence accumulation.

\section{Conclusion}

The usual interpretation of most evidence accumulation models is that the decision processes are preceded and followed by "residual" non-cognitive intervals: $T_{\text {encoding }}$ and $T_{\text {response }}$. The combination of EMG measurement and model fit in a canonical perceptual decision task questions this view. The effects observed on $T_{\text {encoding }}$ and $T_{\text {response }}$ are modified by the participants' strategical adjustments to SAT instructions. This reveals that they reflect more cognitive operations than is commonly assumed. In particular, in the accuracy condition, the model parameters could not be interpreted in terms of the standard theory. The onset of decision does not always reflect the end of encoding, and the onset of the motor response does not always mark the end of the decision. Pending a clarification of this architecture, the interpretation of decision making parameters for scientific or clinical purposes is challenged. Together, our fits and observations show the decisive and constraining role played by non-decision times in the parameter interpretation of decision making models.

\section{Methods}

\section{Participants}

Sixteen participants $(6$ men and 10 women, mean age $=$ 24.5 years, 2 left-handed) that were students at Aix-Marseille University, were recruited for this experiment. All participants reported having normal or corrected vision, and no neurological disorders. The experiment was approved by the ethical experimental committee of Aix-Marseille University, and by the "Comité de Protection des Personnes Sud

${ }^{1}$ When computing the $R^{2}$ on $T_{0}$ estimated from $R T$ rather than from $P M T$ we find the similar results: $R^{2}=0.91$ and $R^{2}=0.88$ in speed, low and high force respectively vs. $R^{2}=-1.11$ and $R^{2}=-0.02$ in accuracy, low and high force. The different levels of adequacy between the V1 data (Reynaud et al., 2012) and DDMs $T_{0}$ were further replicated with a different dataset, see Appendix C. 
Méditerrannée 1" (Approval n 1041). Participants gave their informed written consent, according to the declaration of Helsinki. They received a compensation at a rate of $€ 15$ per hour.

\section{Apparatus}

Participants performed the experiment in a dark and sound-shielded Faraday cage. They were seated in a comfortable chair about $100 \mathrm{~cm}$ away from a 15 inch CRT monitor that had a refresh rate of $75 \mathrm{~Hz}$. The CRT monitor was gamma corrected by a psychophysical procedure provided by the software PsychoPy Peirce (2007). Responses were given by pressing either a left or a right button with the corresponding thumb. The buttons were fixed on top of two cylinders ( 3 $\mathrm{cm}$ in diameter, $7.5 \mathrm{~cm}$ in height). The cylinders were fixed on a tablet and separated by a distance of $20 \mathrm{~cm}$. The buttons were mounted on force sensors that recorded a continuous measure of the force produced at a sampling rate of $2048 \mathrm{~Hz}$. The behavioral response was recorded when a force threshold was exceeded. The device allowed adjusting the force threshold needed for a response to be received. The threshold was manipulated across conditions, as described below. Response signals were transmitted to the parallel port of the recording computer. At button press, participants received a $3 \mathrm{~ms}$ sound feedback (1000 Hz pure tone).

The participants' forearms and hypothenar muscles rested comfortably on the table, to minimize muscle recruitment during response execution. We measured the EMG activity of the flexor pollicis brevis of both hands with two electrodes placed $2 \mathrm{~cm}$ apart on the thenar eminences. This activity was recorded using a BioSemi Active II system (BioSemi Instrumentation, Amsterdam, the Netherlands). The sampling rate was $2048 \mathrm{~Hz}$.

\section{Stimuli}

Stimulus presentation was controlled by the software PsychoPy Peirce (2007). Each stimulus was composed of two vertical oriented gratings, on the left and right of a fixation cross separated by 1.4 visual angle degrees. The gratings had a spatial frequency of 1.2 cycles per visual angle degree and a size of 2.5 visual angle degrees each. At each trial, the same amount of contrast (7\%) was subtracted to the randomly assigned incorrect grating and added to the correct one, resulting in a $14 \%$ contrast difference. The task of the participant was to press the button ipsilateral to the highest contrast.

\section{Experimental manipulations}

Contrast. We choose to manipulate the mean contrast of both gratings while keeping a constant difference of $14 \%$ on a scale between 0 and $100 \%$ (where $0 \%$ is uniform grey) between them. Six levels of mean contrast $(23 \%, 37 \%, 51 \%$,
$65 \%, 79 \%, 83 \%$ ) were selected based on a pilot study, targeting a performance that would typically span from nearperfect accuracy to near chance level. The mean contrast across both patches was randomly chosen at each trial with a fixed rate of occurrence (1/6) within every block.

Force. The Force factor had two levels: strong and weak. These levels were tailored to each participant before the experiment started. Participants were asked to press twice the right and then the left button, with the maximum force they could apply. The maximum voluntary force was defined as the maximum between the two trials from the weakest of the two hands. Defining maximum voluntary force this way was chosen to avoid muscular fatigue from the weakest hand. The actual force levels for the strong and weak conditions were then defined as, respectively, $2 \%$ and $20 \%$ of this maximum voluntary force level (generating force levels around 1.20 and $12 \mathrm{~N}$ respectively).

SAT. The speed-accuracy trade-off (SAT) instruction was manipulated between blocks. Participants were instructed that "Speed" instructions required a mean reaction time near $400 \mathrm{~ms}$ and that "Accuracy" instructions required a percentage of correct responses near $90 \%$ while maintaining RTs below $800 \mathrm{~ms}$. Each block started with the presentation on the center of the screen of its corresponding instruction: the French word for Speed ("Vitesse") or Accuracy ("Précision"). The end of each block was followed by feedback about mean reaction time and mean accuracy, along with oral feedback from the experimenter, if the participant had not satisfied the condition goals of the block.

\section{Procedure}

All participants performed a single experimental session with 24 blocks of 100 trials each. Session duration was around $1 \mathrm{~h} 30$ including a training session of 15 minutes and self-paced breaks between each block. Participants were asked to keep their gaze on the central fixation cross throughout each block, and to respond to the visual stimuli according to the corresponding SAT instruction.

The training session started with 40 trials without specific SAT instructions, followed by 2 blocks of 10 trials in the Speed condition, followed by 2 blocks in Accuracy condition, and ended with 2 blocks of 10 trials with alternating instructions. During the experimental session, SAT instructions alternated every three consecutive blocks. The force settings varied every six blocks, with an on-screen message to inform the participant beforehand. The order of the SAT instructions and the force requirement was counterbalanced across participants so that every possible order combination was presented to 4 participants. Within each block, the 6 levels of mean contrast value were fully randomized across trials. No response deadline was applied, and the inter-trial interval was fixed to $1000 \mathrm{~ms}$ from button press to next stimulus onset. 


\section{EMG processing}

The EMG recordings were read in Python using the MNE module Gramfort et al. (2013). The signal was filtered using a Butterworth 3rd order high pass filter at $10 \mathrm{~Hz}$ from the scipy Python module Oliphant (2007), then segmented by-trial in windows between $150 \mathrm{~ms}$ before and $1500 \mathrm{~ms}$ after stimulus onset. We used a variance-based method to detect whether EMG activity was significantly above threshold in either hands' channels. The precise burst onset was then identified with an algorithm based on the "Integrated Profile" of the EMG burst Liu and Liu (2016); Santello and Mcdonagh (1998). If the algorithm failed to locate or detect the EMG burst onset, the experimenter corrected or added them manually. At this stage of signal processing, the experimenter was unaware of the trial type he was annotating to avoid any bias. Every muscular event (above-threshold change in the signal followed by a return to the baseline) in the trial was marked, even when the activation was not immediately followed by an overt response. In trials where a single EMG burst was detected, motor time $(M T)$ was defined as the time between the onset of EMG burst and the force threshold crossing recorded. Pre-motor time (PMT) was defined as the time between stimulus onset and the EMG burst onset. Multiple EMGs were observed in $21 \%$ of trials. Such observations are not new Weindel et al. (2021), but a precise account of these multiple activities is still lacking. Minimally, they show that participants where not always engaged in a pure sequential encoding-decision-execution process. Therefore, we removed these trials from all the analysis in the study.

\section{Bayesian Statistics}

All analysis were performed in a Bayesian framework. Bayesian methods try to estimate an unknown parameter (or set of parameters) and the uncertainty around it. More explicitly, Bayesian methods combine prior information and Bayes' rule to quantify the likelihood of the parameters by generating a posterior distribution for each of them. This posterior distribution can be naturally interpreted as the probability of any given parameter value given the priors, the data, and the tested model. In our study we summarize the posterior distribution using the mean and the Credible Interval (CrI), the $95 \%$ Highest Probability Density interval or HPD Kruschke (2010).

\section{Hierarchical Modelling}

All models, including linear models, were constrained to follow a hierarchical structure with parameters from each participant as units assumed to be drawn from a population distribution. This parametrization allows to estimate population parameters (e.g., the slope of the effect of stimulus contrast on $R T \mathrm{~s}$ ) along with individual parameters (e.g., the inter-individual differences in the slope of contrast with RT), often referred to respectively as fixed and random effects in the case of linear models. Hierarchical modelling remains Bayesian thus preserving the uncertainties associated with parameter values. Such approach allowed directly testing our hypotheses, by comparing the posterior distributions for the population effects across conditions.

\section{Linear Mixed Models}

We used linear mixed models (LMM) on the log transformed $R T, P M T$, and $M T$, and generalized linear mixed models (gLMM) for the proportion of correct responses. Formally, LMM model the dependent variable as drawn from a normal distribution who's parameters are constrained by the experimental design (e.g. the mean of the normal distribution changes with SAT instructions).

Given our analysis plan, we derived generic LMMs for $R T, P M T$ and $M T$ where all fixed effects and all random effects were estimated. The effects of the experimental factors were modeled on the mean parameter for normally distributed dependent variables (DV), assuming equal variance across conditions.

$$
\begin{gathered}
y_{j i} \sim \mathcal{N}\left(\mu_{j}, \sigma^{2}\right) \\
\mu_{j}=\alpha_{j}+\beta_{1 j} S A T+\beta_{2 j} F C+\beta_{3 j} \text { Con } . \\
+\beta_{12 j} S A T \times F C+\beta_{23 j} S A T \times C o n . \\
+\beta_{13 j} F C \times \text { Con } .+\beta_{123 j} F C \times S A T \times \text { Con } .
\end{gathered}
$$

Where $y_{j i}$ represents the modeled DV (RT, PMT or $\left.M T\right)$ on the $i$ th trial for the $j$ th participant and is assumed to be normally distributed with mean $\mu_{j}$ and standard deviation $\sigma^{2}$. As seen in Equation 2, $\mu$ is dependent of the experimental factors (Con. for mean contrast, FC for Force Condition and SAT for speed accuracy trade-off instructions). The LMM where only fitted on correct responses as Weindel et al. (2021) already reported the effect on these variables on errors and, for the sake of simplicity, to limit the analysis to three factors.

Response correctness was modelled with a gLMM, whereby proportion of correct responses is hypothesized to follow a Bernoulli distribution, modulated by the same factors than in Equation 2:

$$
p\left(\text { response }_{i j}=1\right) \sim \text { Bernoulli }\left(\operatorname{logit}\left(\mu_{j}\right)\right)
$$

To reiterate, in the LMMs and the gLMM, the intercept and all factors and interactions are modeled as random effects :

$$
\begin{gathered}
\alpha_{j} \sim \mathcal{N}\left(\mu_{\alpha}, \sigma_{\alpha}^{2}\right) \\
\beta_{x j} \sim \mathcal{N}\left(\mu_{\beta_{x}}, \sigma_{\beta_{x}}^{2}\right)
\end{gathered}
$$

where $\mu_{\alpha}$ and $\mu_{\beta_{x}}$ are the population estimated intercept and regression coefficient and $\sigma_{\alpha}^{2}$ and $\sigma_{\beta_{x}}^{2}$ the estimated random effect of the population sampling. 
Fitting procedure for the $\mathrm{g} / \mathrm{LMMS}$. For each LMM and gLMM, six Markov Chain Monte Carlo (MCMC) sampling processes were run in parallel, each composed of 2000 iterations among which the first 1000 samples were discarded as warm-up samples. We assessed convergence of the MCMC chains both by computing the potential scale reduction factor $(\hat{R}$, see Gelman, Rubin, et al., 1992) and by means of visual inspection of the MCMC chains. We visually checked the assumptions of the linear regression by inspecting the normality of the residuals through QQ-plots and assessment of homeoscedasticity. The LMM and generalized LMM were fitted with a custom Stan code and using the pystan package Stan Development Team (n.d.). The summary statistics and plots of the parameters were created using arviz python package (version 0.4.1, Kumar, Carroll, Hartikainen, \& Martin, 2019).

Priors for the g/LMMs. The priors for the LMM and gLMM are intended to ease the fitting procedure, we chose to use the relatively broad informative priors described as described in Weindel et al. (2021).

\section{Estimated difference between condition levels $\hat{d}$}

In order to estimate the magnitude of the difference $(\hat{d})$ between the levels of the experimental factors Contrast, Force and SAT, we chose to use the predictions of the fitted linear models. For each dependent variable, we first computed the predicted difference between both SAT level with all other predictors set at 0 (see SI 1). We then computed the predicted differences between the lowest and highest contrast level as well as the weak and high force condition for each SAT instruction separately. The results are thus composed of the effect of SAT, the effects of force and contrast in each SAT condition and the difference of these effects across SAT conditions. Due to the Bayesian nature of the analysis, the uncertainties associated with the regression parameters are preserved in these estimated differences. Thanks to the hierarchical nature of the regression models, we directly estimate a population difference. Both the Bayesian and hierarchical nature of the method therefore allow to directly infer the population level effect size with its uncertainty using the posterior distribution of the predicted difference. The strength of evidence for the presence or absence of an effect was determined based on the credible values of the differences as provided by the mean and the $95 \% \mathrm{CrI}$ of the posterior distribution.

\section{Drift Diffusion Modelling}

Model fitting procedure. We used the implementation of a hierarchical Bayesian DDM provided in the HDDM python package T. V. Wiecki, Sofer, and Frank (2013). Note that $H D D M$ uses the diffusion coefficient Ratcliff and McKoon (2008) as a scaling parameter by fixing it to a value of 1 (contrary to a value of 0.1 in some applications of the
DDM). For each model on $R T$ and on $P M T$, both in the "Model selection" section below and for the model including co-variates, we ran 32500 burn-in samples and 2500 actual recorded samples across four Markov chains Monte-Carlo (MCMC). We inspected each parameter of each chain visually to assess whether they reached their stationary distribution, and whether the $\hat{R}$ Gelman et al. (1992) was under the conventional threshold of 1.01. Additionally, we examined the autocorrelation of each chain to ensure that samples were drawn independently. For the priors, because our design is canonical and in order to ease convergence, we used the default informative priors used in HDDM Matzke and Wagenmakers (2009). Almost all parameters were estimated individually with the constrain of being drawn from a common normal distribution (or half-normal depending on the boundaries, e.g., variability parameters cannot have a negative value). Only the inter-trial variability parameters of the drift rate, of the bias, and of the non-decision time were estimated at the group-level because they are notoriously difficult to estimate Boehm, Annis, et al. (2018); T. Wiecki, Sofer, and Frank (2016).

Model selection. We designed a base model and added parameters according to our hypotheses. The base model was chosen based on previous studies. For this base model, the boundary parameter was free to vary with SAT instructions. The drift rate was free to vary with the contrast ${ }^{2}$, as this parameter has been shown to be associated with stimulus strength. The $T_{0}$ was free to vary with SAT, as it has been observed that this parameter also varies with SAT conditions Palmer et al. (2005); Ratcliff (2006); Voss et al. (2004), with the Force Condition and with the contrast factor, as all three are the factors of interest in the study of $T_{0}{ }^{3}$. The accumulation bias was free to vary for each participant. We also added inter-trial variability of the drift rate and the non-decision time, because of their ability to reduce the influence of contaminant fast-trials Lerche, Voss, and Nagler (2017). Finally, we added the inter-trial variability of the starting point parameter which was free to vary with SAT instructions, because it is often reported that the latency contrast between errors and correct response does change according to the SAT condition and that this pattern is captured by a different bias variability.

In addition to the base model, we tested the following hypothesis, and combinations thereof: whether the drift rate

\footnotetext{
${ }^{2}$ As boundaries were coded as right and left responses (respectively upper and lower threshold), and in order to avoid estimating one drift for each combination of stimulus side and contrast, the model was coded to take negative drift value when the correct stimulus was on the left

${ }^{3}$ We started by including models that do not allow the parameter $T_{0}$ to take different values for the different contrast levels but these models failed to converge probably because of the effects reported in the behavioral results section
} 
also varies with SAT Rae, Heathcote, Donkin, Averell, and Brown (2014), or with the Force Condition Voss et al. (2004); and whether the bias and the boundaries are variable between Force conditions Gomez et al. (2015); Ho et al. (2009); Voss et al. (2004)). The 16 possible combinations of hypotheses are summarized in Table $\mathrm{S} 1$.

We used the deviance information criterion (DIC) to select among competing models. The DIC is an analog to the Akaiake information criterion (AIC) generalized to the hierarchical Bayesian estimation method, in which the improvement of the log-likelihood is weighted against the cost of additional parameters. But because DIC tends to select overfitted models Ando (2007) we also report for each model the Bayesian predictive information criterion Ando (2007). BPIC is intended to correct DIC's bias in favor of over-fitted models by increasing the penalty term for the number of parameters. For all these measures, a lower value of DIC or BPIC indicates a preferred model.

DDM regression analysis. Once the best-fitting model was identified and selected, the effects of the experimental factors on the parameters were assessed by further embedding a hierarchical regression in the model fitting procedure Boehm, Marsman, Matzke, and Wagenmakers (2018). The three experimental factors and their interactions were included as predictors in the regression, on the condition that had been left free to vary across conditions in the model selection procedure. Each parameter that is free to vary with one or more factors was estimated with one intercept and one slope for each factor and interactions. This allowed to use the posterior distribution of intercept and slopes to test directly for the presence and the direction of an effect by inspecting whether 0 is included in the posterior distribution. We compared the results of joint DDM-regression fits on the $P M T$ and $R T$.

The hierarchical nature of the data is preserved in these models because each intercept and slope parameters is estimated as being drawn from a population distribution. The parameters that do not vary with experimental factors (i.e., inter-trial variability of the drift rate and the nondecision time) are estimated as described in the model selection section. The inter-trial variability of the bias was free to vary between SAT instructions but the corresponding effect size was not estimated with a regression. This is because, first, we only have one estimate for the population due to the difficulty to estimate it and, second, we do not have specific hypothesis about this parameter. As in the model selection procedure, the models were fitted using the HDDM python package T. V. Wiecki et al. (2013).

\section{Fast-guess detection and removal}

Fast guess trials can be problematic when studying decision making in the context of evidence accumulation models. Before performing any statistical analysis, we applied an exponentially weighted moving average filter or EWMA Vandekerckhove and Tuerlinckx (2007). This method iteratively computes a weighted accuracy measure (amount of correct responses relative to errors) from the fastest to the slowest response time. The method is usually performed on the sorted $R T$ distribution, but was applied here to the sorted $P M T$ distribution. Participants are considered to be in a fast guess state until the weighted accuracy is higher than a defined threshold. The $P M T$ at which this change of state occurs is identified, and faster trials are censored. EWMA involves the following user-defined parameters: the initial starting point of the weighted accuracy, the amount of preceding trials (weight) retained in the accuracy computation, and the accuracy threshold for defining non-guess trials. The starting point was defined at 0.50 based on the assumption that a guessing strategy yields a $50 \%$ chance of correct response. The weight (bounded from 0 to 1 , with 0 being all preceding trials used) was fixed at 0.01 as in the description of the method Vandekerckhove and Tuerlinckx (2007). The threshold was fixed at 0.55 based on a reasonable assumption that participants could not reach an accuracy superior to 0.55 on the basis of guessing.

The EWMA filter was applied for each participant's PMT distribution, separately in the speed and accuracy conditions; fast-guesses can have different latencies across both conditions. $P M T$ s rather that $R T$ s were used for the EWMA, first because previous application of the method showed a high reliability when the method was applied separately to $P M T$ and $R T$ Weindel et al. (2021), and second because trials that do not appear very fast on $R T$ can sometimes be fast on the $P M T$ and therefore be problematic when fitting a DDM on the $P M T$ as done here. The figures illustrating these rejection procedures can be found in the online repository.

\section{Acknowledgments}

The authors thank Iván Ballasch for his help in data collection, Anna Montagnini, Mathieu Servant, and Thierry Hasbroucq for fruitful discussions, Frédéric Chavane and Alexandre Reynaud for help with the data from their article.

\section{References}

Ando, T. (2007). Bayesian predictive information criterion for the evaluation of hierarchical bayesian and empirical bayes models. Biometrika, 94(2), 443-458.

Barthélemy, F. V., Fleuriet, J., \& Masson, G. S. (2010). Temporal dynamics of $2 \mathrm{~d}$ motion integration for ocular following in macaque monkeys. Journal of neurophysiology, 103(3), 1275-1282.

Boehm, U., Annis, J., Frank, M. J., Hawkins, G. E., Heathcote, A., Kellen, D., ... Wagenmakers, E.-J. (2018). Estimating Between-Trial Variability Parameters of the Diffusion Decision Model: Expert Advice and Recommendations. PsyArXiv. doi: 10.17605/OSF.IO/KM28U 
Boehm, U., Marsman, M., Matzke, D., \& Wagenmakers, E.-J. (2018). On the importance of avoiding shortcuts in applying cognitive models to hierarchical data. Behavior research methods, 50(4), 1614-1631.

Brown, S., \& Heathcote, A. (2005). A ballistic model of choice response time. Psychological review, 112(1), 117-128. doi: 10.1037/0033-295X.112.1.117

Buc Calderon, C., Dewulf, M., Gevers, W., \& Verguts, T. (2017). Continuous track paths reveal additive evidence integration in multistep decision making. Proceedings of the $\mathrm{Na}$ tional Academy of Sciences, 114(40), 10618-10623. doi: 10.1073/pnas.1710913114

Burle, B., Possamaï, C.-A., Vidal, F., Bonnet, M., \& Hasbroucq, T. (2002, nov). Executive control in the Simon effect: an electromyographic and distributional analysis. Psychological research, 66(4), 324-36. doi: 10.1007/s00426-002-0105-6

Burle, B., Roger, C., Vidal, F., \& Hasbroucq, T. (2008). Spatiotemporal dynamics of information processing in the brain: Recent advances, current limitations and future challenges. International Journal of Bioelectromagnetism, 10, 17-21.

Cisek, P., Puskas, G. A., \& El-Murr, S. (2009). Decisions in changing conditions: the urgency-gating model. Journal of Neuroscience, 29(37), 11560-11571.

Donders, F. C. (1868). Die schnelligkeit psychischer processe: Erster artikel. Archiv für Anatomie, Physiologie und wissenschaftliche Medicin, 657-681.

Dutilh, G., Annis, J., Brown, S. D., Cassey, P., Evans, N. J., Grasman, R. P., ... others (2016). The quality of response time data inference: A blinded, collaborative assessment of the validity of cognitive models. Psychonomic bulletin $\mathcal{E}$ review, $1-19$.

Dutilh, G., Wagenmakers, E.-J., Visser, I., \& van der Maas, H. L. (2011). A phase transition model for the speed-accuracy trade-off in response time experiments. Cognitive Science, $35(2), 211-250$.

Gelman, A., Rubin, D. B., et al. (1992). Inference from iterative simulation using multiple sequences. Statistical science, 7(4), 457-472.

Gomez, P., Ratcliff, R., \& Childers, R. (2015). Pointing, looking at, and pressing keys: A diffusion model account of response modality. Journal of Experimental Psychology: Human Perception and Performance, 41(6), 1515-1523. doi: 10.1037/a0039653

Gramfort, A., Luessi, M., Larson, E., Engemann, D. A., Strohmeier, D., Brodbeck, C., ... Hämäläinen, M. (2013). MEG and EEG data analysis with MNE-Python. Frontiers in Neuroscience(7 DEC). doi: 10.3389/fnins.2013.00267

Harwerth, R. S., \& Levi, D. M. (1978). Reaction time as a measure of suprathreshold grating detection. Vision research, 18(11), $1579-1586$.

Heathcote, A., \& Love, J. (2012). Linear deterministic accumulator models of simple choice. Frontiers in Psychology, 3(AUG), 1-19. doi: 10.3389/fpsyg.2012.00292

Ho, T. C., Brown, S., \& Serences, J. T. (2009). Domain general mechanisms of perceptual decision making in human cortex. Journal of Neuroscience, 29(27), 8675-8687.

Kruschke, J. K. (2010). Bayesian data analysis. Wiley Interdisciplinary Reviews: Cognitive Science, 1(5), 658-676.
Kumar, R., Carroll, C., Hartikainen, A., \& Martin, O. A. (2019). ArviZ a unified library for exploratory analysis of Bayesian models in Python. The Journal of Open Source Software. Retrieved from http://joss. theoj .org/papers/10.21105/joss.01143 doi: 10.21105 /joss.01143

Lerche, V., Voss, A., \& Nagler, M. (2017). How many trials are required for parameter estimation in diffusion modeling? A comparison of different optimization criteria. Behavior Research Methods, 49(2), 513-537. doi: 10.3758/s13428-0160740-2

Liu, J., \& Liu, Q. (2016, feb). Use of the integrated profile for voluntary muscle activity detection using EMG signals with spurious background spikes: A study with incomplete spinal cord injury. Biomedical Signal Processing and Control, 24, 19-24. doi: 10.1016/j.bspc.2015.09.004

Luce, R. (1986). Response Times: Their Role in Inferring Elementary Mental Organization. $O x$ ford University Press New York(3), 562. doi: 10.1093/acprof:oso/9780195070019.001.0001

Matzke, D., \& Wagenmakers, E.-J. (2009). Psychological interpretation of the ex-gaussian and shifted wald parameters: A diffusion model analysis. Psychonomic bulletin $\mathcal{E}$ review, 16(5), 798-817.

Naka, K., \& Rushton, W. A. (1966). S-potentials from colour units in the retina of fish (cyprinidae). The Journal of physiology, 185(3), 536-555.

Oliphant, T. E. (2007). SciPy: Open source scientific tools for Python. Computing in Science and Engineering, 9, 10-20. Retrieved from http://www.scipy.org/ doi: 10.1109/MCSE.2007.58

Ollman, R. (1966). Fast guesses in choice reaction time. Psychonomic Science, 6(4), 155-156.

Oriet, C., \& Jolicœur, P. (2003). Absence of perceptual processing during reconfiguration of task set. Journal of Experimental Psychology: Human Perception and Performance, 29(5), 1036.

Palmer, J., Huk, A. C., \& Shadlen, M. N. (2005). The effect of stimulus strength on the speed and accuracy of a perceptual decision. Journal of Vision, 5(5), 1. doi: 10.1167/5.5.1

Pashler, H. (1994). Dual-task interference in simple tasks: data and theory. Psychological Bulletin, 116(2), 220.

Peirce, J. W. (2007). PsychoPy-Psychophysics software in Python. Journal of Neuroscience Methods, 162(1-2), 8-13. doi: 10.1016/j.jneumeth.2006.11.017

Rae, B., Heathcote, A., Donkin, C., Averell, L., \& Brown, S. (2014). The Hare and the Tortoise: Emphasizing speed can change the evidence used to make decisions. Journal of Experimental Psychology: Learning, Memory, and Cognition, 40(5), 1226-1243. doi: 10.1037/a0036801

Ratcliff, R. (1978). A theory of memory retrieval. Psychological Review, 85(2), 59-108. doi: 10.1037/0033-295X.85.2.59

Ratcliff, R. (2006). Modeling response signal and response time data. Cognitive psychology, 53(3), 195-237.

Ratcliff, R., \& McKoon, G. (2008). The diffusion decision model: theory and data for two-choice decision tasks. Neural computation, 20(4), 873-922. doi: 10.1162/neco.2008.12-06420 
Ratcliff, R., \& McKoon, G. (2018). Modeling numerosity representation with an integrated diffusion model. Psychological review, 125(2), 183.

Ratcliff, R., \& Rouder, J. N. (1998). Modeling response times for two-choice decisions. Psychological Science, 9(5), 347356.

Ratcliff, R., Smith, P. L., Brown, S. D., \& McKoon, G. (2016). Diffusion Decision Model: Current Issues and History. Trends in Cognitive Sciences, 20(4), 260-281. doi: $10.1016 /$ j.tics.2016.01.007

Ratcliff, R., \& Tuerlinckx, F. (2002a). Estimating parameters of the diffusion model: approaches to dealing with contaminant reaction times and parameter variability. Psychonomic bulletin $\mathcal{E}$ review, 9(3), 438-481. doi: 10.3758/BF03196302

Ratcliff, R., \& Tuerlinckx, F. (2002b). Estimating parameters of the diffusion model: Approaches to dealing with contaminant reaction times and parameter variability. Psychonomic bulletin $\mathcal{G}$ review, 9(3), 438-481.

Resulaj, A., Kiani, R., Wolpert, D. M., \& Shadlen, M. N. (2009). Changes of mind in decision-making. Nature, 461(7261), 263-266. doi: 10.1038/nature 08275

Reynaud, A., Masson, G. S., \& Chavane, F. (2012). Dynamics of local input normalization result from balanced short-and long-range intracortical interactions in area v1. Journal of neuroscience, 32(36), 12558-12569.

Santello, M., \& Mcdonagh, M. J. (1998). The control of timing and amplitude of EMG activity in landing movements in humans. Experimental Physiology, 83(6), 857-874. doi: 10.1113/expphysiol.1998.sp004165

Scaltritti, M., Job, R., Alario, F.-X., \& Sulpizio, S. (2020). On the boundaries between decision and action: Effector-selective lateralization of beta-frequency power is modulated by the lexical frequency of printed words. Journal of Cognitive Neuroscience, 32(11), 2131-2144.

Schmidgen, H. (2002). Of frogs and men: the origins of psychophysiological time experiments, 1850-1865. Endeavour, 26(4), 142 - 148. doi: https://doi.org/10.1016/S01609327(02)01466-7

Selen, L. P. J., Shadlen, M. N., \& Wolpert, D. M. (2012). Deliberation in the Motor System: Reflex Gains Track Evolving Evidence Leading to a Decision. Journal of Neuroscience, 32(7), 2276-2286. doi: 10.1523/JNEUROSCI.527311.2012

Servant, M., Logan, G. D., Gajdos, T., \& Evans, N. J. (2021). An integrated theory of deciding and acting. Journal of Experimental Psychology: General.

Servant, M., White, C., Montagnini, A., \& Burle, B. (2015). Using Covert Response Activation to Test Latent Assumptions of Formal Decision-Making Models in Humans. Journal of Neuroscience, 35(28), 10371-10385. doi: 10.1523/JNEUROSCI.0078-15.2015

Servant, M., White, C., Montagnini, A., \& Burle, B. (2016, oct). Linking Theoretical Decision-making Mechanisms in the Simon Task with Electrophysiological Data: A Model-based Neuroscience Study in Humans. Journal of Cognitive Neuroscience, 28(10), 1501-1521. doi: 10.1162/jocna00989

Smith, P. L., \& Lilburn, S. D. (2020). Vision for the blind: visual psychophysics and blinded inference for decision mod- els. Psychonomic Bulletin $\mathcal{E}$ Review.

Spieser, L., Servant, M., Hasbroucq, T., \& Burle, B. (2017, jun). Beyond decision! Motor contribution to speed-accuracy trade-off in decision-making. Psychonomic Bulletin $\mathcal{E}$ Review, 24(3), 950-956. doi: 10.3758/s13423-016-1172-9

Stan Development Team. (n.d.). Pystan: the python interface to stan. Retrieved from http://mc-stan.org

Steinemann, N. A., O'Connell, R. G., \& Kelly, S. P. (2018). Decisions are expedited through multiple neural adjustments spanning the sensorimotor hierarchy. Nature communications, 9(1), 3627.

Stevens, S. S. (1961). To honor fechner and repeal his law. Science, 133(3446), 80-86.

Stine, G. M., Zylberberg, A., Ditterich, J., \& Shadlen, M. N. (2020). Differentiating between integration and nonintegration strategies in perceptual decision making. Elife, 9, e55365.

Stone, M. (1960). Models for choice-reaction time. Psychometrika, 25(3), 251-260. doi: 10.1007/BF02289729

Usher, M., \& McClelland, J. L. (2001). The time course of perceptual choice: The leaky, competing accumulator model. Psychological Review, 108(3), 550-592. doi: 10.1037//0033295X.108.3.550

Vandekerckhove, J., \& Tuerlinckx, F. (2007). Fitting the ratcliff diffusion model to experimental data. Psychonomic bulletin $\mathcal{E}$ review, 14(6), 1011-1026.

van Maanen, L., Portoles, O., \& Borst, J. P. (2021). The discovery and interpretation of evidence accumulation stages. Computational Brain $\mathcal{F}$ Behavior, 1-21.

Verdonck, S., Loossens, T., \& Philiastides, M. G. (2021). The leaky integrating threshold and its impact on evidence accumulation models of choice response time (rt). Psychological Review, 128(2), 203.

Voss, A., Rothermund, K., \& Voss, J. (2004). Interpreting the parameters of the diffusion model: an empirical validation. Memory $\mathcal{E}$ cognition, 32(7), 1206-1220. doi: 10.3758/BF03196893

Weindel, G., Anders, R., Alario, F.-X., \& Burle, B. (2021). Assessing model-based inferences in decision making with singletrial response time decomposition. Journal of Experimental Psychology: General.

Wiecki, T., Sofer, I., \& Frank, M. (2016). Hddm 0.6. 0 documentation.

Wiecki, T. V., Sofer, I., \& Frank, M. J. (2013). Hddm: Hierarchical bayesian estimation of the drift-diffusion model in python. Frontiers in neuroinformatics, 7, 14.

\section{Appendix A}

From Linear model parameters to estimated effects

All regression models, including the regression on the DDM parameters followed the same factor coding scheme. The SAT factor was coded as a treatment factor ( 0 for accuracy and 1 for speed). The force condition was coded as a sum contrast (-0.5 for weak and 0.5 for strong force). The factor contrast was centered on the middle value and scaled so that -0.5 represented the lowest contrast and 0.5 the highest. A summary of this factor coding for the regression models is 
given in the matrices below, the first row represent the original levels, the second row the values on which the regression were estimated :

$$
\begin{aligned}
& S A T=\left(\begin{array}{cc}
\text { Accuracy } & \text { Speed } \\
0 & 1
\end{array}\right) \\
& \text { Force }=\left(\begin{array}{cc}
\text { Weak } & \text { Strong } \\
-0.5 & 0.5
\end{array}\right) \\
& \text { Contrast }=\left(\begin{array}{cccccc}
23 \% & 37 \% & 51 \% & 65 \% & 79 \% & 93 \% \\
-.5 & -.3 & -.1 & .1 & .3 & .5
\end{array}\right)
\end{aligned}
$$

These coding features were chosen to ease the interpretation of the resultant coefficients. When the binary predictor is sum-contrasted ( -0.5 and 0.5$)$, the estimated $\beta$ value can be read as the difference between both conditions. When the binary predictor is treatment-contrasted ( 0 and 1$)$, the estimated $\beta$ can be read as the difference to add to the intercept (predictor at 0 ) to obtain the mean of the condition where the predictor is at value 1 . Hence, in our analysis, the intercept can be read as the predicted time for the reference condition where the SAT emphasis is on accuracy, and at an intermediate value for the predictors contrast and $\mathrm{FC}$.

Given these coding features and the Bayesian nature of the estimation we can estimate the effect of a factor in a given condition and preserve the uncertainty associated with the effects. E.g. To compute the effect of Force in the speed condition we can add the interaction term $\beta_{S A T \times F o r c e}$ to the estimated $\beta_{\text {Force }}$ in the accuracy condition. As coefficients are estimated using a MCMC procedure this addition is done on each MCMC iteration, allowing to keep the uncertainty around the resulting coefficient.

Units of the $\mathbf{g} /$ LMMs parameters. For the LMMs on proportion correct, $R T, P M T$ and $M T$, the data was transformed prior to the modeling (logit for proportion correct and $\log$ for the other variables). Using Monte Carlo Markov Chain (MCMC) processes, we back-transformed the predictions of the linear models for the chosen differences at each iteration, with the exponential for log transformed variables (LMM) or the inverse logit for proportion correct (gLMM). This preserves the uncertainty around the parameter values while reverting them to the natural units of the dependent variables.

The full procedure for performing the differences can be found in the online repository https://osf.io/t2ar3/.

\section{Appendix B}

Model Selection

As seen in table B1 the DIC criterion almost always favor the complex models over the simpler one. However two patterns are consistent across the models, allowing the boundaries and the bias to vary with force conditions and drift rate to vary with SAT in addition to the contrast always improves the goodness of fit as assessed by the DIC. However when considering the BPIC criterion, initially intended to correct the complexity bias of the DIC, only allowing the variation of force on boundaries and on the bias seems to improve the goodness of fit. Hence based on BPIC we select the model allowing the boundaries and the bias to vary across force condition in addition to the designed base model (respectively M13 and M1 in Table B1). Importantly the results of the model selection is the same for a fit on PMT. The goodness of fit both on RT and PMT as displayed with quantile probability plot (see Figure B2 and B1) is satisfactory in most conditions but the amount of errors is rather misfitted when considering a high force especially in the fit on PMT (that pattern is common across all tested models).

\section{Appendix C \\ Replication of RT DDM results}

In a follow-up study targeting encoding processes with electro-encephalography, we designed an experiment close to the present one. The only major differences are that three contrast levels $(23,51$ and $83 \%)$ were sub-sampled from the six levels used in the main experiment, and that force was alternated every three block instead of every six block. To test for the replicability of the results on DDM modelling of the RTs we ran the same analysis, as described in the method section, on the new dataset obtained from 20 participants. The results appear in table $\mathrm{C} 1$.

Effects on $T_{0}$. As in the original study, estimated $T_{0}$ was longer when accuracy was emphasized and when a high force was required. Contrast and SAT interacted showing evidence for a negative relationship between contrast and $T_{0}$ in speed, but not in accuracy. We found no evidence for the two way interaction Force and Contrast, as well as the three way interaction of Force, Contrast and SAT. However, contrary to the original study, we observe that Force and SAT did not interact.

Effects on decision related parameters. As previously, the boundary parameter was affected by SAT, being smaller for the speed condition, and it was not reliably affected by Force in either SAT condition. The starting point parameter revealed no evidence for an effect of Force.

Replication of the link between estimated encoding processes and V1 data. As in the original analysis presented in the discussion section, we again observe a high coherence between V1 data and encoding processes as estimated with $T_{0}$ on the new dataset but only in the speed condition. The computed $R^{2}$ was high in the speed condition both with high and low force, respectively $R^{2}=0.92$ and $R^{2}=0.53$, but lower in the accuracy condition, $R^{2}=0.44$ and $R^{2}=0.21$.

Overall all results presented for the fit of a DDM on RT replicate in the new sample except the interaction between SAT and Force that was found to be absent in the repli- 

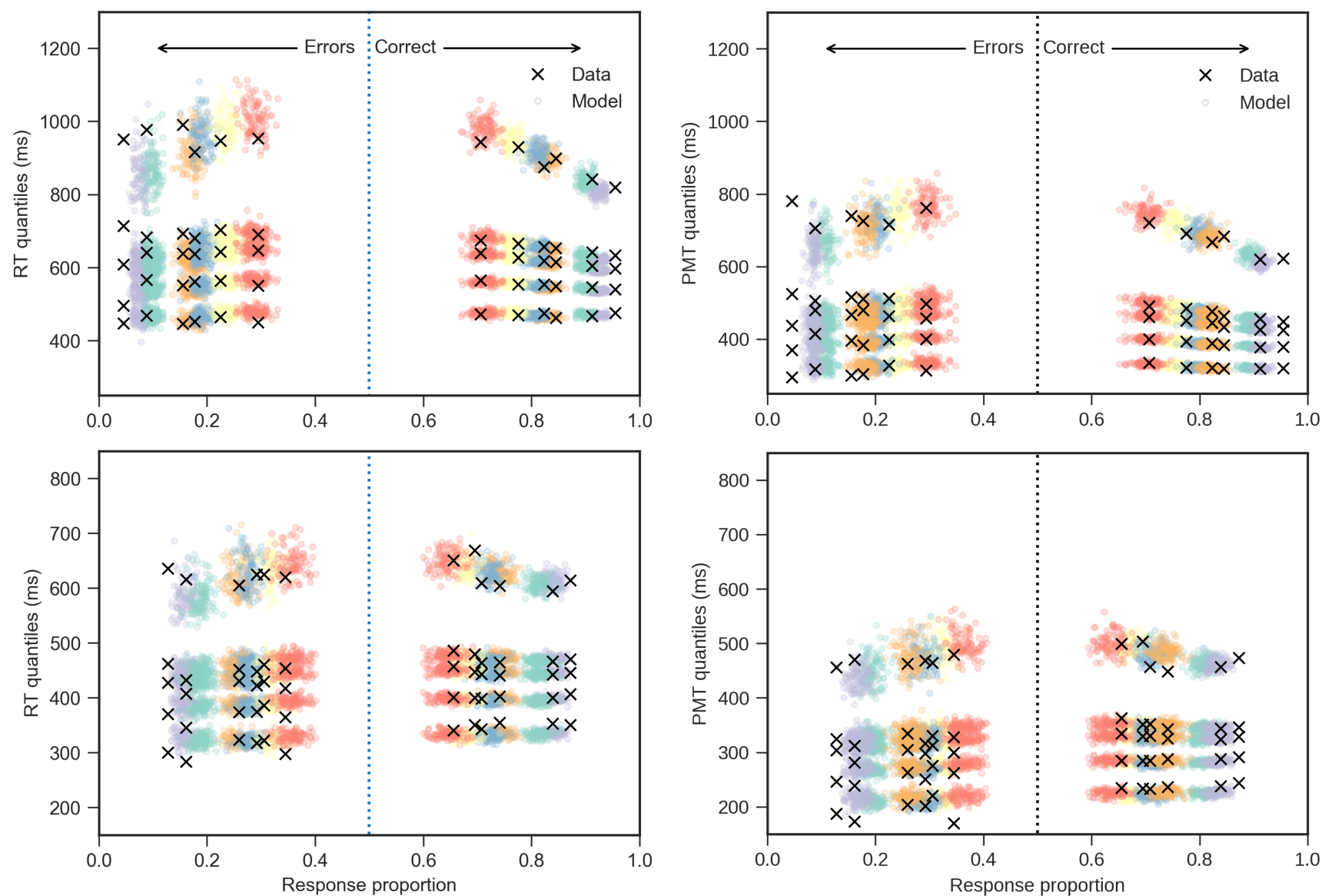

Figure B1. Quantile-probability plots (Ratcliff \& McKoon, 2008) for high force based on a fit on RT (left column) and PMT (right column), in the accuracy (upper row) and speed (lower row) conditions, computed from the best fitting model.

The X-axis displays obtained response proportion across contrast levels (color coded), symmetrically for errors (left side) and correct responses (right side). The Y-axis displays the fitted (dot) and observed (cross) $R T$ binned in 5 quantiles (.1, .3, .5, .7 and .9 quantiles, from bottom to top). Observed response proportion and $R T$ quantiles were computed from values pooled across participants. Model predictions were obtained by drawing 250 parameter values from the joint posterior distribution and computing their associated predicted performance. The misfit of the DDM is particularly apparent in the fit on PMT in accuracy (upper right corner), where the DDM clearly predicts a response proportion lower than the one observed on the data.

cation. We do not have a specific interpretation for this lack of replication. One possibility is that the increased alternance between force conditions (from every six block in the original study, to every three block) might have changed the link between both blocked factors.

\section{Appendix D}

Predictions by V1 neuron activation onset

Reynaud et al. (2012) performed a measurement of the temporal activation of V1 neurons in awake monkey using voltage sensitive dye and the variation of this temporal activation with the contrast of stimuli close to the one used in this study. They then fitted the relationship between onset of V1 neurons activity and contrast with an inverted Naka-rushton equation from Barthélemy et al. (2010) :

$$
\tau_{c}(c)=\tau_{\max }+\tau_{\text {shift }} \cdot \frac{c_{n}}{c_{n}+s_{50}^{n}}
$$

Where $c$ is contrast, $\tau_{\max }$ and $\tau_{\text {shift }}$ are respectively the minimum latency observed at highest contrast and the maximum decrease in latency. $n$ is the estimated latency shift exponent, $s_{50}$ the estimated half decay contrast value. For the purpose of our analysis we recovered the values of the parameters estimated by Reynaud et al. (2012) and draw the predictions associated with the mean contrast levels used in our study.

Figure D1 represents the adjustment between point estimate of $T_{0}$ and $T_{\text {encoding }}$ with the curve predicted by the recovered parameters of Reynaud et al. (2012) for the inverted Naka-Rushton equation. However, since monkey latencies are shorter than those we observed with humans, we 

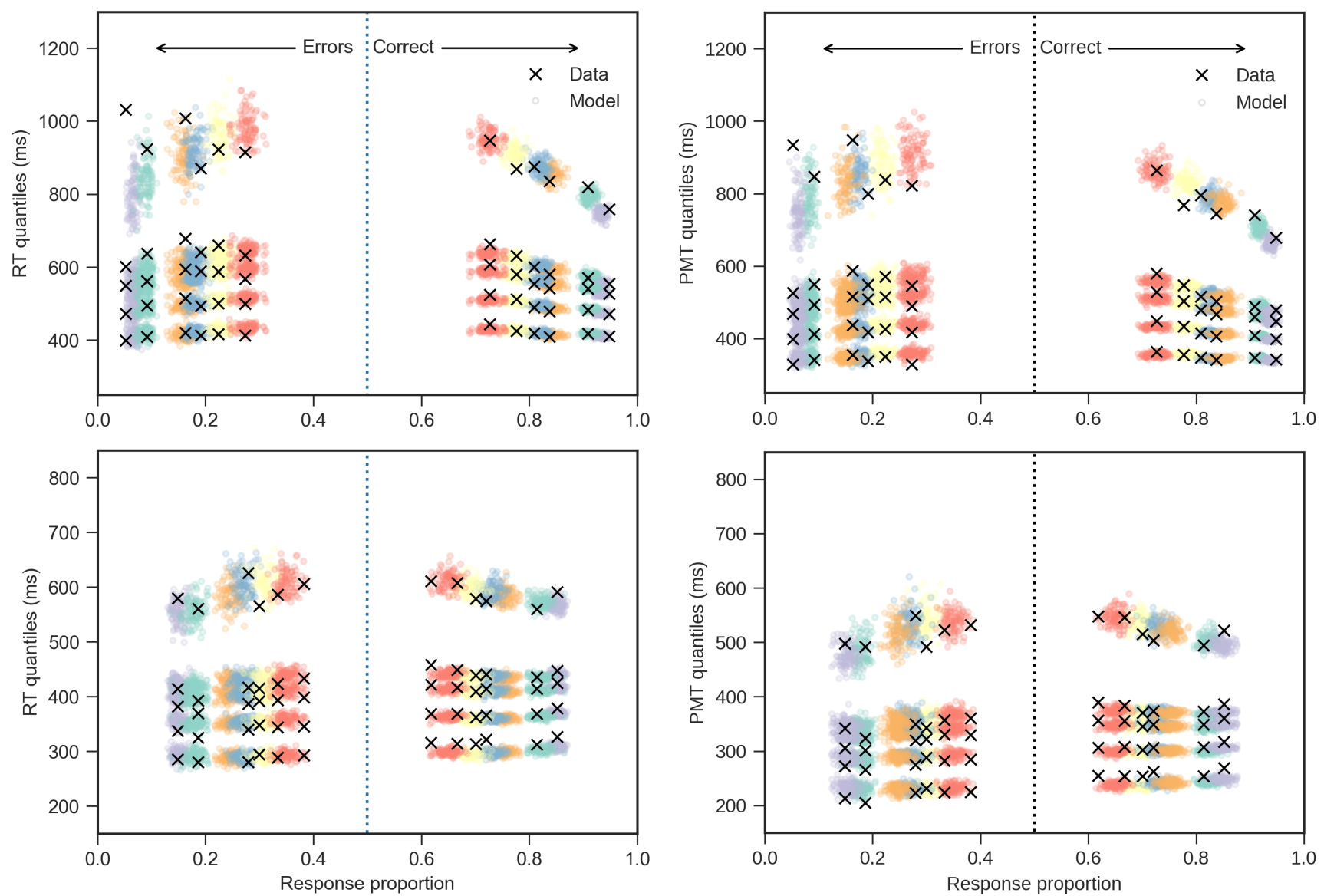

Figure B2. Quantile-probability plots (Ratcliff \& McKoon, 2008) for low force based on a fit on RT (left column) and PMT (right column), in the accuracy (upper row) and speed (lower row) conditions, computed from the best fitting model.

The misfit of the DDM is particularly apparent in the fit on PMT in accuracy (upper right corner), where the DDM clearly predicts a response proportion lower than the one observed on the data.

centered them by subtracting their mean and adding the mean

pants.

of the $T_{\text {encoding }}$ computed over all contrast levels and partici- 

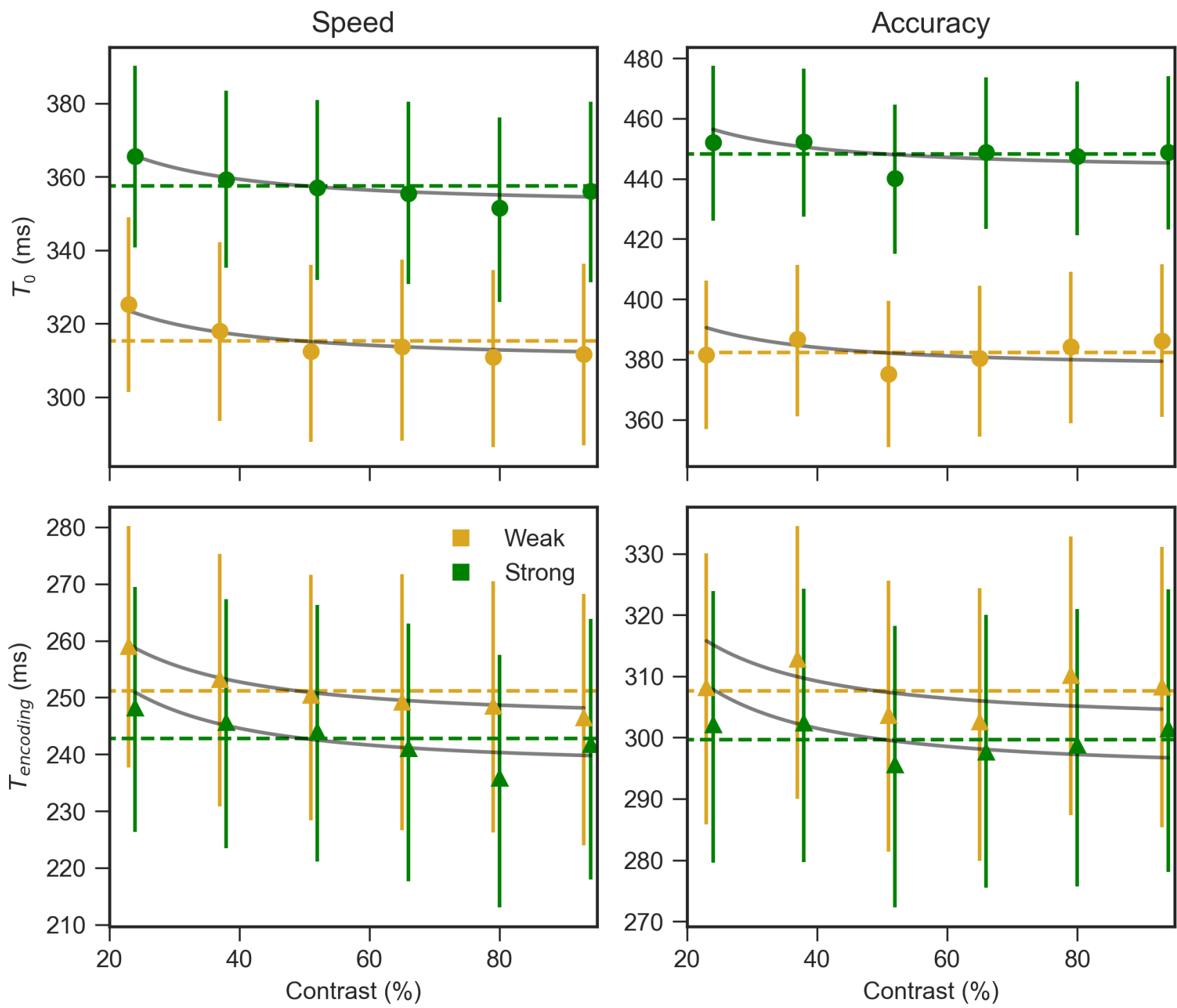

Figure D1. Estimated $T_{0}$ (obtained from a DDM fit on $R T$ ) and $T_{\text {encoding }}$ (obtained from a fit on $P M T$ ) across contrast levels and splitted between SAT and Force conditions. Bars around the point estimate represents $65 \% \mathrm{CrI}$ of the population mean. The colored lines represent the mean of $T_{0}$ or $T_{\text {encoding }}$ for each corresponding sub-cell. The grey lines represents the values predicted by the parameters of the inverted Naka-Rushton recovered from Reynaud, Masson, and Chavane 2012. These prediction have been first centered on 0 by subtracting their mean then rescaled by adding the mean of the corresponding sub-cell. Grey and color lines have therefore the same mean in each sub-cell formed by the combination of SAT and Force levels. 
Table 1

\begin{tabular}{|c|c|c|c|c|c|c|c|}
\hline \multirow[b]{2}{*}{ DDM Par. } & & & RT & & & PMT & \\
\hline & Factor & $\hat{d}$ & $2.5 \%$ & $97.5 \%$ & $\hat{d}$ & $2.5 \%$ & $97.5 \%$ \\
\hline \multirow{2}{*}{ Drift } & (Intercept) & 1.79 & 2.03 & 1.51 & 1.91 & 2.19 & 1.62 \\
\hline & Contrast & -1.77 & -1.46 & -2.08 & -1.90 & -1.55 & -2.23 \\
\hline \multirow{5}{*}{ Boundaries } & (Intercept) & 1.08 & 1.02 & 1.15 & 1.05 & 0.99 & 1.10 \\
\hline & SAT & -0.33 & -0.40 & -0.25 & -0.30 & -0.38 & -0.22 \\
\hline & FC:Acc. & -0.06 & -0.14 & 0.02 & -0.17 & -0.26 & -0.08 \\
\hline & FC:Speed & -0.03 & -0.13 & 0.08 & -0.07 & -0.19 & 0.05 \\
\hline & FC:Speed-Acc. & 0.03 & -0.05 & 0.11 & 0.10 & 0.01 & 0.18 \\
\hline \multirow{2}{*}{ Bias } & (Intercept) & 0.50 & 0.48 & 0.53 & 0.49 & 0.47 & 0.51 \\
\hline & $\mathrm{FC}$ & 0.004 & -0.001 & 0.01 & 0.002 & -0.002 & 0.006 \\
\hline \multirow{11}{*}{$T_{0}(\mathrm{~ms})$} & (Intercept) & 414.6 & 391.0 & 439.1 & 301.2 & 280.0 & 322.8 \\
\hline & SAT & -77.7 & -106.1 & -49.0 & -51.5 & -77.7 & -22.7 \\
\hline & FC:Acc. & 70.6 & 54.1 & 87.2 & -4.2 & -14.6 & 6.6 \\
\hline & FC:Speed & 46.3 & 26.0 & 66.2 & -5.6 & -19.7 & 9.1 \\
\hline & FC:Speed-Acc. & -24.3 & -37.2 & -11.3 & -1.4 & -12.4 & 9.8 \\
\hline & Contrast:Acc. & 1.0 & -8.8 & 11.0 & 0.0 & -9.7 & 9.4 \\
\hline & Contrast:Speed & -10.2 & -21.6 & 0.7 & -8.9 & -20.0 & 2.0 \\
\hline & Contrast:Speed-Acc. & -11.2 & -19.3 & -2.7 & -8.8 & -16.6 & -1.1 \\
\hline & $\mathrm{FC} \times$ Contrast:Acc. & -6.0 & -16.0 & 4.1 & -2.5 & -11.9 & 6.8 \\
\hline & FC $\times$ Contrast:Speed & 5.5 & -3.6 & 14.7 & 6.9 & -1.6 & 15.5 \\
\hline & $\mathrm{FC} \times$ Contrast:Speed-Acc. & 11.5 & -1.3 & 24.7 & 9.3 & -4.1 & 21.5 \\
\hline
\end{tabular}

Comparison of Estimated differences between conditions levels $(\hat{d})$ across fits on RT and on PMT. Columns labelled $\hat{d}$ refer to the maxima a posteriori from the corresponding marginal posterior distribution. Columns labelled $2.5 \%$ and $97.5 \%$ refer to the CrI intervals. Bold and italic fonts refer to the sign of the estimate, bold for positive values, italic for negative values. A replication of the $R T$ results is provided in the Appendix $C$ (Table C1). 


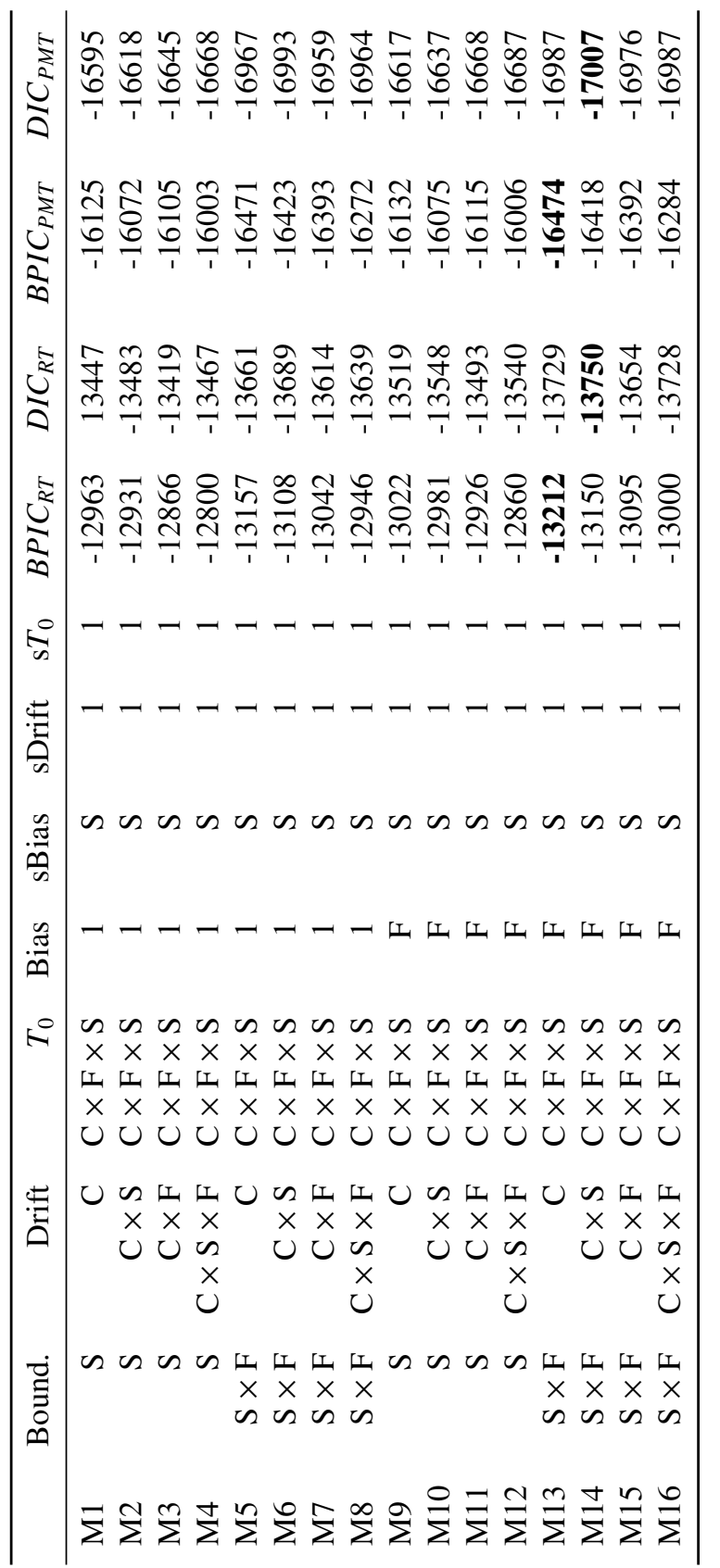

초 인

iे 
Table C1

\begin{tabular}{|l|l|c|c|c|}
\hline \multirow{3}{*}{ DDM Par. } & \multicolumn{1}{|c|}{ Factor } & & RT & \\
\cline { 2 - 5 } Drift & (Intercept) & $\hat{d}$ & $2.5 \%$ & $97.5 \%$ \\
\hline \multirow{5}{*}{ Boundaries } & Contrast & $\mathbf{1 . 8 5}$ & $\mathbf{2 . 0 5}$ & $\mathbf{1 . 6 6}$ \\
& (Intercept) & -1.01 & -0.66 & -1.36 \\
\cline { 2 - 5 } & SAT & $\mathbf{1 . 1 6}$ & $\mathbf{1 . 0 9}$ & $\mathbf{1 . 2 3}$ \\
& FC:Acc. & -0.42 & -0.49 & -0.34 \\
& FC:Speed & -0.03 & -0.12 & $\mathbf{0 . 0 5}$ \\
& FC:Speed-Acc. & $\mathbf{0 . 0 2}$ & -0.09 & $\mathbf{0 . 1 5}$ \\
\hline \multirow{5}{*}{ Bias } & (Intercept) & $\mathbf{0 . 0 5}$ & -0.04 & $\mathbf{0 . 1 5}$ \\
\hline & FC & $\mathbf{0 . 0 0 3}$ & -0.001 & $\mathbf{0 . 0 0 7}$ \\
\hline \multirow{5}{*}{$(m s)$} & (Intercept) & $\mathbf{4 0 0 . 1}$ & $\mathbf{3 7 6 . 7}$ & $\mathbf{4 2 5 . 4}$ \\
& SAT & -67.8 & -83.2 & -49.2 \\
& FC:Acc. & $\mathbf{4 4 . 4}$ & $\mathbf{2 5 . 7}$ & $\mathbf{6 4 . 6}$ \\
& FC:Speed & $\mathbf{4 3 . 5}$ & $\mathbf{1 5 . 9}$ & $\mathbf{6 8 . 8}$ \\
& FC:Speed-Acc. & -0.9 & -20.5 & $\mathbf{1 9 . 4}$ \\
& Contrast:Acc. & -3.1 & -9.0 & $\mathbf{2 . 6}$ \\
& Contrast:Speed & -10.8 & -17.6 & -4.4 \\
& Contrast:Speed-Acc. & -7.7 & -14.5 & -1.3 \\
& FC $\times$ Contrast:Acc. & $\mathbf{0 . 9}$ & -7.2 & $\mathbf{9 . 9}$ \\
& FC $\times$ Contrast:Speed & $\mathbf{6 . 3}$ & -5.3 & $\mathbf{1 7 . 3}$ \\
& FC $\times$ Contrast:Speed-Acc. & $\mathbf{5 . 4}$ & -8.1 & $\mathbf{1 9 . 4}$ \\
\hline
\end{tabular}

Comparison of Estimated differences $(\hat{d})$ between conditions levels for a fit on RT on a replication dataset. Column labelled $\hat{d}$ refer to the maxima a posteriori from the corresponding marginal posterior distribution. Columns labelled $2.5 \%$ and $97.5 \%$ refer to the CrI intervals. Bold and italic fonts refer to the sign of the estimate, bold for positive values, italic for negative values. 